\title{
Eliciting ambiguity aversion in unknown and in compound lotteries: a smooth ambiguity model experimental study
}

\author{
Giuseppe Attanasi • Christian Gollier • \\ Aldo Montesano • Noemi Pace
}

(C) Springer Science+Business Media New York 2014

\begin{abstract}
Coherent-ambiguity aversion is defined within the (Klibanoff et al., Econometrica 73:1849-1892, 2005) smooth-ambiguity model (henceforth $K M M$ ) as the combination of choice-ambiguity and value-ambiguity aversion. Five ambiguous decision tasks are analyzed theoretically, where an individual faces two-stage lotteries with binomial, uniform, or unknown second-order probabilities. Theoretical predictions are then tested through a 10-task experiment. In (unambiguous) tasks 1-5, risk aversion is elicited through both a portfolio choice method and a BDM mechanism. In (ambiguous) tasks 6-10, choice-ambiguity aversion is elicited through the portfolio choice method, while value-ambiguity aversion comes about through the $B D M$ mechanism. The behavior of over $75 \%$ of classified subjects is in line with the KMM model in all tasks 6-10, independent of their degree of risk aversion. Furthermore, the percentage of coherent-ambiguity-averse subjects is lower in the binomial than in the uniform and in the unknown treatments, with only the latter difference being significant. The most part of coherent-ambiguity-loving subjects show a high risk aversion.
\end{abstract}

Keywords Coherent-ambiguity aversion - Value-ambiguity aversion .

Choice-ambiguity aversion - Smooth ambiguity model - Binomial distribution . Uniform distribution · Unknown urn

JEL Classification D81 - D83 · C91

G. Attanasi (凶)

University of Strasbourg (BETA), Strasbourg, France

e-mail: attanasi@unistra.fr

C. Gollier

Toulouse School of Economics, Toulouse, France

A. Montesano

Bocconi University, Milan, Italy

N. Pace

University Ca' Foscari of Venice, Venice, Italy 


\section{Introduction}

This paper proposes a series of ten experimental decision tasks involving two-outcome lottery choices. Five of these tasks are aimed at eliciting a subject's attitude toward risk, and the other five are designed to study her attitude toward ambiguity. Specific theoretical predictions about a subject's behavior in the latter decision tasks are obtained, by relying on the Klibanoff et al. (2005) smooth ambiguity model (henceforth $K M M$ ). The paper has three main goals.

The first objective is to propose a simple experimental method able to make $K M M$ operational in individual decision tasks. To this purpose, the experimental environment is explicitly designed in order to match $K M M$ intuition of modeling ambiguity through two-stage lotteries. In such an environment, two different operational definitions of ambiguity aversion are provided. The first one, namely value-ambiguity attitude, is based on Becker and Brownson (1964) idea that "individuals are willing to pay money to avoid actions involving ambiguity" (p. 5). ${ }^{1}$ A value-ambiguity-averse subject values an ambiguous lottery less than its unambiguous equivalent with the same mean probabilities. In the $K M M$ model, this is true if the subject's $\phi$ function is concave. The second definition, namely choice-ambiguity attitude, relies on Gollier (2012) intuition that more ambiguity-averse subjects should have a smaller demand for a risky asset whose distribution of returns is ambiguous. It should be noted that a portfolio containing a larger share invested in the risky asset may be seen as a two-stage lottery where second-order objective probabilities are more dispersed. In the $K M M$ framework, Gollier (2012) has shown that an ambiguity-averse subject might have a larger demand for the risky asset than another ambiguity-neutral subject with the same risk aversion, thereby deducting that a value-ambiguity averse subject is not necessarily choice-ambiguity averse. On the other hand, Gollier (2012) provides a set of sufficient conditions on the structure of the two-stage uncertainty to re-establish the link between the concavity of $\phi$ and choice-ambiguity aversion. Given that one of these sufficient conditions is satisfied in our experimental decision tasks, an equivalence between value-ambiguity attitude and choice-ambiguity attitude is expected, and defined as coherent-ambiguity attitude within the KMM framework.

The second objective of the paper is to check behavioral predictions obtained within the $K M M$ model in the five decision tasks aimed at studying a subject's ambiguity attitude. In all ambiguous decision tasks, the subject always faces the same two (secondstage) lottery outcomes. Thus, within the same treatment, each ambiguous task differs from the next one only because of the level of ambiguity of the decision setting and/or

\footnotetext{
1 After Becker and Brownson (1964), the idea that information which reduces ambiguity has a positive value for ambiguity-averse subjects has been clearly stated within different decision-theoretic models, e.g., Quiggin (2007), using Machina (2004) concept of almost-objective acts; Attanasi and Montesano (2012), relying on the Choquet expected utility model. Moreover, focusing on a specific adaptation of $K M M$, Snow (2010) has proved that the value of information that resolves ambiguity increases with greater ambiguity and with greater ambiguity aversion. Attanasi and Montesano (2012) have obtained similar results within the Choquet model.
} 
because of a first-degree stochastic improvement in the distribution of second-order probabilities. In particular, these tasks are designed in such a way that once a subject has been classified as coherent-ambiguity-averse, coherent-ambiguity-neutral, or coherent-ambiguity-loving, the sign of the variation of her certainty equivalent from one task to the next one should depend only on this classification. Therefore, this sign should be predicted directly by the "sign" of her attitude toward ambiguity as determined within $K M M$. This means that, by construction, the verification of our main theoretical predictions in these tasks should be independent of the subject's degree of risk aversion as elicited in the five unambiguous tasks. Finding an effect of risk attitude over the behavioral verification of our theoretical hints would raise some doubts on the use of $K M M$ as reference model for the tasks proposed in our experiment. The elicitation of risk attitude is also important in order to empirically ascertain whether it influences the "sign" of the ambiguity attitude, i.e., which one of the three ambiguity attitudes (aversion, neutrality, or proneness) the subject will show. Our design is also aimed at finding whether this "sign" may depend on the riskiness of the second-stage lottery, i.e., on the spread of the difference of its two outcomes. In order to be consistent in the elicitation of risk attitude and of ambiguity attitude, the same pair of instruments are used for both attitudes. In particular, risk attitude is elicited through both a portfolio choice method and a Becker et al. (1964) mechanism (henceforth $B D M)$. Correspondingly, choice-ambiguity aversion is established through the first method and value-ambiguity aversion through the second one. The combination of the two instruments has a twofold role. For risk attitude, it allows to check that both instruments lead to similar subjects' orderings. For ambiguity attitude, it affords to elicit separately the two features of (coherent)-ambiguity attitude introduced above within $K M M$. Concerning risk attitude, once the correlation between the two riskaversion orderings has been verified, the results of the portfolio choice method are relied upon: this has the advantage of imposing some theoretically derived constraints which allow to check whether the subject's selected portfolio is compatible with a constant absolute and/or a constant relative risk aversion specification. Concerning ambiguity attitude, throughout the paper we consider as "classified subjects" only those who provide coherent answers under the two instruments. This provides a rationale for the term "coherent" to identify the kind of ambiguity attitude studied in this paper.

The third objective of the paper is to analyze how subjects' decisions under ambiguity react to different distributions of second-order probabilities. The experiment consists of three treatments, according to a between-subject design. The five unambiguous tasks do not vary among treatments, while the ambiguous tasks are different for each treatment, in the way in which uncertainty over the composition of the urns used to perform them is generated. More precisely, the first of these tasks relies on a 10-ball small urn containing white and orange balls; subjects are not told its composition. In all treatments said composition is generated through a random draw from a big urn, introduced in order to mimic KMM two-stage lottery approach. In treatment 1 , the composition of the 10-ball small urn is determined through a Bernoullian process over a 50-white-50-orange balls big urn, thereby leading to a binomial distribution of second-order probabilities. In treatment 2 , subjects are shown that second-order probabilities over the composition of the 10-ball small urn are uniformly distributed. 
In treatment 3, subjects have no information about the composition of the 10-ball small urn, although - to make it comparable to treatment 1 - ambiguity is generated through a two-stage lottery procedure similar to the one of the binomial treatment, though with no information provided about the composition of the big urn. The uniform distribution of the second-order probabilities in treatment 2 is clearly a mean-preserving spread of the binomial distribution obtained in treatment 1 . Treatment 3 is intrinsically more ambiguous than treatment 1 . Therefore, under ambiguity aversion, it is expected that in the first ambiguous task of both the uniform and the unknown treatment, the subject will assign a lower value to the ambiguous lottery than in the corresponding task of the binomial treatment. This should also happen in the remaining ambiguous tasks, provided, once the 10-ball small urn is generated, the way its composition is "modified" in order to vary the level of ambiguity and the distribution of second-order probabilities is the same in each treatment. Although our design is not within-subject, the abovestated predictions can be checked by comparing the distribution of subjects' decisions in the ambiguous tasks of the three treatments. This treatment comparison would hold only under the assumption that the distribution of subjects' degree of risk aversion does not differ among the three treatments. This is an additional reason for eliciting risk attitude before looking at subjects' decisions in the ambiguous tasks.

The rest of the article is structured as follows. Section 2 describes the experimental design, by highlighting the motivations behind the ten decision tasks. Section 3 analyzes the five decision tasks under ambiguity and presents the main theoretical results. Section 4 presents the results of our experiment. In Sect. 5, the experimental design and results are discussed within the experimental literature on ambiguity aversion. Section 6 concludes.

\section{Experimental design}

Experimental subjects were graduate students in Economics of the Toulouse School of Economics (TSE). Computerized sessions were conducted at the Laboratory of Experimental Economics of TSE. A total of 105 (42 women, 63 men, average age $=23.70$ ) participated in our experiment, with each subject participating only once. Average earnings were approximately $€ 20.50$ per subject, including a $€ 5.00$ show-up fee. The experiment was programed using the z-Tree software (Fischbacher 2007) and subjects were seated in isolated cubicles in front of computer terminals. Three treatments were run through a between-subject design, with the same number of subjects $(N=35)$ participating in each treatment. The number of subjects in each session varied from a minimum of 9 to a maximum of $18 .^{2}$

The experiment consists of ten decision tasks per treatment. ${ }^{3}$ At the beginning of the experiment, participants were told the number of tasks. However, instructions for every new task were given and read aloud only prior to that task. After instructions

\footnotetext{
2 For treatment 1, two sessions were run, respectively, with 17 and 18 students. Treatment 2 had three sessions, respectively, with 16,10, and 9 students. Treatment 3 also had three sessions, respectively, with 12,10 , and 13 subjects.

3 Experimental instructions are available upon request.
} 
Table 1 Main features of the ten decision tasks

\begin{tabular}{|c|c|c|c|c|c|}
\hline & All Treatments & Treatment 1 & Treatment 2 & Treatment 3 & \\
\hline Task & Elicitation Method & \multicolumn{3}{|c|}{ Features of the lotteries } & Task \\
\hline $1-4$ & Portfolio Choice & \multirow{2}{*}{\multicolumn{3}{|c|}{ Simple Lottery }} & $1-4$ \\
\hline 5 & $B D M$ mechanism & & & & 5 \\
\hline $6-9$ & $B D M$ mechanism & \multirow{2}{*}{$\begin{array}{c}\text { Binomial } \\
\text { Compound Lottery }\end{array}$} & \multirow{2}{*}{$\begin{array}{c}\text { Uniform } \\
\text { Compound Lottery }\end{array}$} & \multirow{2}{*}{$\begin{array}{c}\text { Unknown } \\
\text { (Compound) Lottery }\end{array}$} & $6-9$ \\
\hline 10 & Portfolio Choice & & & & 10 \\
\hline
\end{tabular}

Table 2 Portfolio choice in tasks 1-4: pairs of lottery outcomes

\begin{tabular}{|c|c|c|c|c|c|c|c|c|}
\hline & \multicolumn{2}{|c|}{ Task $t=1$} & \multicolumn{2}{|c|}{ Task $t=2$} & \multicolumn{2}{|c|}{ Task $t=3$} & \multicolumn{2}{|c|}{ Task $t=4$} \\
\hline & $\bar{x}_{1}^{j}$ & $\underline{x}_{1}^{j}$ & $\bar{x}_{2}^{j}$ & $\underline{x}_{2}^{j}$ & $\bar{x}_{3}^{j}$ & $\underline{x}_{3}^{j}$ & $\bar{x}_{4}^{j}$ & $\underline{x}_{4}^{j}$ \\
\hline Lottery $j=A$ & 12 & 6 & 11 & 6 & 20 & 14 & 19 & 14 \\
\hline Lottery $j=B$ & 16 & 4 & 14 & 4 & 24 & 12 & 22 & 12 \\
\hline Lottery $j=C$ & 20 & 2 & 17 & 2 & 28 & 10 & 28 & 8 \\
\hline Lottery $j=D$ & 24 & 0 & 20 & 0 & 32 & 8 & 34 & 4 \\
\hline
\end{tabular}

were read aloud, the decision task appeared on the screen, and participants had 3 minutes to answer the task. The average duration of the experiment was 65 minutes, including construction of the "unknown" small urns (only for treatment 1 and 3), performance of one over the ten tasks and participants' final payment. The final payment of each participant depended only on the choice made by this participant in the ten decision tasks and on some random draws which are explained furth er below. Only one of the ten decision tasks was randomly selected at the end of the experiment to determine participants' final earnings.

The ten tasks in the experimental design differ in terms of the elicitation method applied and/or of the scope of that elicitation (see Table 1). Tasks 1-5 do not vary among treatments, while tasks 6-10 are different for each treatment in the way in which uncertainty over the composition of the urns is generated.

In each task from 1 to 4 , the experimental subject is shown the same transparent small urn with 5 white balls and 5 orange balls inside. She is asked to choose among four simple lotteries of the type $l_{t}^{j}=\left(\bar{x}_{t}^{j}, 0.5 ; \underline{x}_{t}^{j}, 0.5\right)$, with $\bar{x}_{t}^{j}, \underline{x}_{t}^{j} \in \mathbb{R}_{+}, \bar{x}_{t}^{j}>\underline{x}_{t}^{j}$ for each $j$ and $t$, where $j=A, B, C, D$ indicates the four lotteries in each task and $t=1,2,3,4$ indicates the task (see Table 2). All $l_{t}^{j}$ in the four tasks rely on the same 5-5 balls small urn, with white balls assigned to the highest of the two outcomes, $\bar{x}_{t}^{j}$. Each $l_{t}^{j}$ differs from the other fifteen lotteries proposed in the four tasks in terms of both expected value and standard deviation. In particular, in each of the four portfolio choices, the higher the index $j$ of the lottery, the higher both its expected value and its standard deviation (see Table 7 in the Appendix). Let $j_{t} \in\{A, B, C, D\}$ be the index of the lottery chosen by the subject in task $t \in\{1,2,3,4\}$. If a task $t$ between 1 and 4 is selected to be paid at the end of the experiment, the subject plays for the pair of outcomes she has chosen in that task, namely $\bar{x}_{t}^{j_{t}}$ and $\underline{x}_{t}^{j_{t}}$. She is paid $\bar{x}_{t}^{j_{t}}$ if a white ball is randomly drawn from the 5-5 balls small urn and $\underline{x}_{t}^{j_{t}}$ otherwise.

Tasks 1-4 are called "portfolio choices" because the random outcome parallels the outcome of a portfolio with one risk-free asset and one risky asset. Indeed, the outcome 
G. Attanasi et al.

Table 3 Reinterpretation of the lottery choices into portfolio choices for tasks $1-4$

\begin{tabular}{lrrrrrrl}
\hline & $w_{t}$ & $\bar{y}_{t}$ & $\underline{y}_{t}$ & $\alpha_{t}^{j=A}$ & $\alpha_{t}^{j=B}$ & $\alpha_{t}^{j=C}$ & $\alpha_{t}^{j=D}$ \\
\hline Task $t=1$ & 8 & 4 & -2 & 1 & 2 & 3 & 4 \\
Task $t=2$ & 8 & 3 & -2 & 1 & 2 & 3 & 4 \\
Task $t=3$ & 16 & 4 & -2 & 1 & 2 & 3 & 4 \\
Task $t=4$ & 16 & 3 & -2 & 1 & 2 & 4 & 6 \\
\hline
\end{tabular}

$l_{t}^{j t}$ of choice $j$ in task $t$ can be written as $\left(w_{t}-\alpha_{t}^{j}\right)\left(1+r_{f}\right)+\alpha_{t}^{j}\left(1+\widetilde{y}_{t}\right)$, where $w_{t}$ can be interpreted as the initial wealth in task $t$, and $\alpha_{t}^{j}$ is the euro investment in the risky asset: $r_{f}$ is the risk-free rate that is always normalized to 0 , and $\widetilde{y}_{t}$ is the return of the risky asset in task $t$. The return of the risky asset can take two possible values $\bar{y}_{t}$ and $\underline{y}_{t}$ with equal probabilities. In Table 3 , the portfolio contexts and portfolio choices in the four tasks are reinterpreted.

In task 5, the subject is assigned the same pair of lottery outcomes she has chosen in task 4, namely $\bar{x}_{4}^{j_{4}}$ and $\underline{x}_{4}^{j_{4}}$. The same 5-5 balls small urn of tasks 1-4 is used, with white balls again assigned to $\bar{x}_{4}^{j_{4}}$, in order to build the lottery $l_{4}^{j_{4}}=\left(\bar{x}_{4}^{j_{4}}, 0.5 ; \underline{x}_{4}^{j_{4}}, 0.5\right)$. Therefore, the subject's "initial endowment" in task 5 is her preferred lottery in task 4. In task 5 , the subject has the possibility to sell $l_{4}^{j_{4}}$ through a $B D M$ mechanism. ${ }^{4}$ She is asked to state the smallest price at which she is willing to sell $l_{4}^{j_{4}}$, by setting a price between $x_{4}^{j_{4}}$ and $\bar{x}_{4}^{j_{4}}$. This reservation price should provide an approximation of the subject's certainty equivalent of $l_{4}^{j_{4}}$. The $B D M$ mechanism here used is very close to the one implemented by Halevy (2007). Differently from Halevy (2007), however, there are four lotteries $l_{4}^{j}$ for which a subject may state her reservation price and the set of possible "buying/selling prices" is discrete. The discreteness of the set of possible "buying/selling prices" is due to the fact that the $B D M$ mechanism used here is implemented through real tools, as every instrument in our experimental design. Indeed, as will be shown below, in this experiment none of the random draws from any urn is computerized. In the same spirit of concreteness, for each lottery $l_{4}^{j_{4}}$ chosen in task 4, there is a different envelope containing a finite set of numbered tickets. A random draw of a numbered ticket from this envelope gives the buying price for lottery $l_{4}^{j_{4}} \cdot 5$

\footnotetext{
4 Given that the subject has to set the price at which to sell a random "initial endowment", she is assigned a lottery that she has just declared to prefer among four possible lotteries (task 4). Therefore, her "initial endowment" in task 5 (and, as will be shown, in tasks 6-9) depends on the choice made in task 4, although the subject does not know this in task 4.

5 More specifically, there are four different envelopes, labeled respectively with letter $A, B, C$ and $D$, i.e., one for each lottery available in task 4 . Each of these envelopes contains eleven different numbered tickets. The distance between two subsequent numbers on the tickets in an envelope is the same, so as to have the same number of tickets in each envelope, with the lowest numbered ticket being equal to $\underline{x}_{4}^{j}$ and the highest being equal to $\bar{x}_{4}^{j}$. In particular, the eleven tickets inside envelope $A$ are $14,14.5, \ldots, 18.5,19$; those inside envelope $B$ are $12,13, \ldots, 21,22$; those inside envelope $C$ are $8,10, \ldots, 26,28$; those inside envelope $D$ are $4,7, \ldots, 31,34$. The eleven tickets in envelope $j$ represent the set of possible prices of lottery $l_{4}^{j_{4}}$, with $j=A, B, C, D$. A ticket is randomly drawn from each envelope. The ticket drawn from envelope $j$ determines the random "buying price" for lottery $j$. Then, without knowing this price, the subject states
} 
In each of the tasks 6-9, the subject is also assigned the same pair of lottery outcomes that she has chosen in task 4 , with white balls again assigned to $\bar{x}_{4}^{j_{4}}$, and she is given the possibility to sell the respective lottery through the same $B D M$ mechanism as in task 5. In particular, the subject is told that, in each task from 6 to 9, the "buying prices" for the four lotteries are, respectively, the same four numbered tickets randomly drawn at the beginning of task 5. Therefore, albeit unknown to the subject, the reference "buying price" for the assigned lottery is the same in tasks 5-9. Furthermore, the set of possible selling prices for each lottery is also maintained constant throughout tasks 5-9. However, the 10-ball small urn used to determine the likelihood of $\bar{x}_{4}^{j_{4}}$ and of $\underline{x}_{4}^{j_{4}}$ in tasks 6-9 is not the same as in tasks $1-5$.

Indeed, the three treatments differ in the way in which the composition of the 10ball small urn used to perform task 6 is determined. In Fig. 1 below, implementation of task 6 in each of the three treatments is described. More specifically:

- Treatment 1: Binomial. The 10-ball small urn used to perform task 6 is generated from a transparent big urn containing 50 white balls and 50 orange balls. At the beginning of task 6, 10 balls are randomly drawn (one after the other, with replacement) from the big urn. The colors of these 10 balls determine the composition of the 10-ball small urn. The outcomes of the 10 random draws are not shown to the subject. Therefore, at the moment when the subject states her reservation price in task 6, the composition of the unknown small urn is a binomial random variable taking 11 possible values.

- Treatment 2: Uniform. At the beginning of task 6, each subject is shown a transparent construction urn ${ }^{6}$ containing 11 transparent small urns of 10 balls each. Each of the 11 small urns has a different composition in terms of white and orange balls. One of the 11 small urns would be randomly drawn from the construction urn. Therefore, at the moment when the subject states her reservation price, the composition of the unknown small urn is a (discrete) uniform random variable taking 11 possible values.

- Treatment 3: Unknown. The 10-ball small urn used to perform task 6 is generated from an opaque big urn containing 100 white and orange balls with unknown composition. As in treatment 1, at the beginning of task 6, 10 balls whose color determines the composition of the 10-ball small urn are randomly drawn (one after the other, with replacement) from the big urn. The outcomes of the 10 random draws are not shown to the subject. Therefore, the subject states her reservation price in task 6 without having any information about the composition of the unknown small

\footnotetext{
Footnote 5 continued
}

her smallest selling price (reservation price) for her lottery $l_{4}^{j_{4}}$, by choosing one among the eleven possible prices for lottery $j$. In case task 5 is selected for payment at the end of the experiment, the following happens: if, for the lottery the subject owned in task 5, the subject's smallest selling price is lower than the respective random "buying price", the subject sells her lottery and is paid the latter price. Otherwise, she will have to play her lottery, and her payoff $\left(\bar{x}_{4}^{j_{4}}\right.$ or $\left.\underline{x}_{4}^{j_{4}}\right)$ will depend on the ball randomly drawn from the small urn.

6 The term "construction urn" is borrowed from Klibanoff et al. (2012). Epstein (2010) calls this "secondorder urn". 


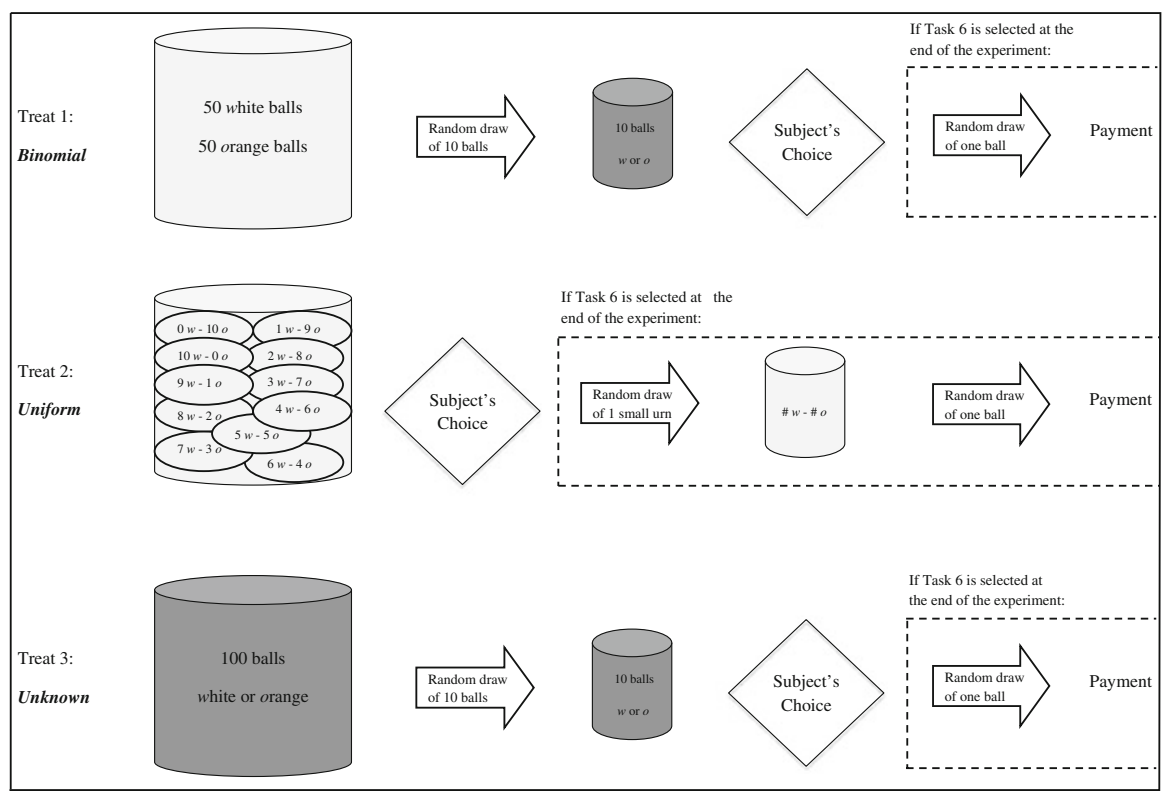

Fig. 1 Implementation of task 6 in each treatment

urn. The reason why ambiguity is generated through a two-stage lottery is to make this treatment comparable to treatment 1.

Tasks 7-9 involve the elimination of some possible compositions of the 10-ball unknown small urn used to perform task 6 . At the beginning of task 7, the subject is told that, if this task were to be performed at the end of the experiment, the number of white balls in the unknown small urn would be between 3 and 7 (and so the number of orange balls). This would be implemented in the following way. In treatment 1 and treatment 3, 6 balls will be taken out of the unknown small urn constructed at the beginning of task 6 and replaced with 3 white balls and 3 orange balls. In treatment 2, six transparent small urns (the three with less than 3 white balls and the three with less than 3 orange balls) will be taken out of the transparent construction urn. Task 8 (9) differs from task 7 only for the fact that in the unknown small urn, the number of white (orange) balls will be between 3 and 10 .

In each of the tasks 6-9 the subject, besides stating her reservation price for the lottery resulting from the corresponding unknown small urn, is also asked to guess the number of white balls in that urn. In case a task from 6 to 9 is randomly selected to be performed at the end of the experiment, the subject is paid an additional $€ 5.00$ if her guess of the number of white balls in the unknown small urn of that task is correct.

Finally, task 10 is the same as task 4 in terms of the elicitation method (portfolio choice) and in the set of possible pairs of outcomes among which the subject has to pick one. However, the 10-ball small urn used to determine the likelihood of the chosen pair, namely $\bar{x}_{10}^{j_{10}}$ and $\underline{x}_{10}^{j_{10}}$, is the same unknown small urn as in task 6. 
It should be noted that the subject in each task has no feedback about any random draw performed in any of the previous tasks. ${ }^{7}$ This is because only one of the tasks is selected and actually performed and only at the end of the experiment. ${ }^{8}$ Therefore, in our experimental design, the subject cannot make any updating either about the actual composition of the unknown small urns or about the random "buying prices" in the $B D M$ mechanism. Also, in each session, all the urns are real urns (not computerized), and all the random draws in the experiment (construction of the small urns, random "buying prices" in the $B D M$ mechanism, selection of the task determining a subject's final earnings, performance of this task) are executed by one of the subjects (indicated in the experimental instructions as the "drawer"). This subject is randomly chosen before the beginning of the experiment among the subjects showing up for the experimental session. He/she does not participate in the experiment and is paid a fix amount of money (\$20.00) independent of his/her random draws. The reason why we opted for a human random "drawer" instead of computerized random draws is to make participants in the experiment aware that no manipulation from the experimenter is possible in any of the random draws characterizing the experimental setting. This was especially important for the random composition of the unknown small urn used for task 6 and the following tasks. ${ }^{9}$ Indeed, the physical implementation of all the

\footnotetext{
7 The ten decision tasks are shown to the subject always in the same order. The reason why tasks $1-5$ (which rely on the 5-5 balls small urn) are proposed always before tasks 6-10 is to elicit the subject's riskaversion before introducing unknown/multiple small urns. About task 5 coming before tasks 6-9, Halevy (2007) has shown that the (usually) higher reservation price for the 5-5 balls small urn (task 5 here) is not a consequence of this urn being proposed before the unknown/multiple ones (tasks 6-9 here). Finally, about the order of tasks 6-9, our theoretical results for the subject's reservation price in tasks $t=6, \ldots, 9$ do not suggest that this price should be always increasing or always decreasing with $t$. Rather, the trend of the subject's reservation price over tasks 6-9 should depend on the "sign" of her attitude toward ambiguity (e.g., see (5) and (7) below). A similar argument holds for task 4 always coming before task 10: different signs of the ambiguity attitude lead to different predictions about if and how the subject's choice varies between the two tasks.

${ }^{8}$ For tasks 1-4 and 10, performing the task means playing the chosen lottery (random draw of one ball from the 10-ball small urn). For tasks 5-9, it means playing the assigned lottery only if the subject's selling price is not lower than the random "buying price" for that lottery.
}

${ }^{9}$ More specifically, in treatment $\mathbf{1}$, the drawer is given the chance to check (in front of all experimental subjects in the session) that the number of white and orange balls in the transparent big urn is 50-50. Then, together with the transparent big urn, he/she is brought by the experimenter behind a screen where he/she performs the random draw of 10 balls (one after the other, with replacement) from the big urn. The screen being inside the laboratory, experimental subjects can "listen" to the random draw but they cannot see the color of the ten randomly drawn balls. After each of the ten random draws, the drawer shows the ball to the experimenter, records its color on a paper sheet, and puts the ball back in the urn. At the end of the ten random draws, the drawer puts 10 balls in an opaque small urn according to the colors recorded on the paper sheet, comes out from behind the screen and shows the opaque small urn to all experimental subjects in the session (they are informed about this procedure before it takes place). Then, he/she places the opaque small urn on a table in front of all experimental subjects and task 6 begins. At the end of the experiment, if task 6 is randomly selected (by the drawer him/herself) to determine participants' final earnings, the drawer randomly draws one of the 10 balls from the opaque small urn. If the randomly selected task is one among tasks $7-9$, the drawer will eliminate some possible compositions of the 10-ball opaque small urn (according to the rules specified above) before randomly drawing one of the 10 balls. The procedure in treatment 3 is as in treatment 1 apart from two features. First, the big urn is opaque and neither the drawer nor any experimental subject in the session may check the number of white and orange balls in the opaque big urn (although this urn is shaken by the drawer in front of everybody to show that there are many balls inside). Second, before the beginning of the experiment the opaque big urn is placed on a table in 
procedures described in Fig. 1 for each treatment was necessary to guarantee that the experimenter cannot (and cannot be seen to) manipulate the implementation of the "ambiguity" device and that the information available about this device is the same for all subjects.

\section{Theoretical predictions}

We use Klibanoff et al. (2005) smooth ambiguity model (henceforth $K M M$ ) as a general framework. Therefore, we assume that the subject's preferences are represented by the von Neumann-Morgenstern Expected Utility (henceforth $E U$ ) function for simple lotteries, and we relax reduction between first and second-order probabilities in two-stage lotteries in order to account for multiplicity/uncertainty of the possible compositions of the second-stage lottery.

The following are our predictions about the subject's behavior in the first half of the experimental design, i.e., in the five tasks aimed at estimating her degree of risk aversion. These five tasks only involve simple lotteries.

Tasks 1-4 rest on the well-known result in expected utility theory (e.g., Pratt 1964) that the value of a simple lottery decreases if the subject's risk aversion increases. The value of a simple lottery $l$ with possible returns $X$ is measured by its certainty equivalent $C E(l)$, which is defined by the following condition:

$$
u(C E(l))=E U(X)
$$

where it is assumed that the utility function $u$ is increasing and that it is concave for risk-averse subject and convex for risk-loving ones. From the previous relation, it derives that $C E(l)$ decreases if the concavity of $u$ increases in the sense of ArrowPratt. This implies that, for any task $1-4$, an increase in risk aversion will never induce the subject to select a riskier lottery (in our case, a lottery with more exposure to the risky asset). Given the fact that lotteries $A, B, C, D$ correspond to different portfolios with an increasing exposure to the risky asset, it is also known from Arrow (1964) that preferences are unimodal in $(A, B, C, D)$. Thus, if for example $C$ is preferred to $B$, it is also the case that it is preferred to $A$. If one limits the analysis to a set of

\footnotetext{
Footnote 9 continued

front of all experimental subjects and the drawer makes a preliminary random draw from a transparent 2-ball urn containing 1 white ball and 1 orange ball. The color of the randomly drawn ball is assigned to the highest of the two outcomes in each lottery in all the ten tasks of the experiment. Then, before the beginning of task 6 the drawer uses the opaque big urn to determine the composition of the 10-ball opaque small urn, according to the same random draw procedure of treatment 1. In treatment 2 the drawer is given the chance to check (in front of all experimental subjects in the session) the composition of each of the 11 transparent small urns inside the transparent construction urn. Then, he/she places this big "urn of all urns" on a table in front of all experimental subjects and task 6 begins. At the end of the experiment, if task 6 is randomly selected (by the drawer him/herself) to determine participants' final earnings, the drawer will first randomly draw one of the 11 transparent small urns from the transparent big urn and then randomly draws one of the 10 balls from this small urn. If the randomly selected task is one among tasks 7-9, the drawer will take out of the transparent big urn some of the 11 transparent small urns (according to the rules specified above) before randomly drawing one of the remaining ones from the transparent big urn.
} 
Table 4 Optimal answers for tasks 1-4 under CARA

\begin{tabular}{|c|c|c|c|c|c|c|c|}
\hline \multicolumn{3}{|c|}{ Predicted pattern under $C A R A$} & \multicolumn{5}{|c|}{ Experimental Data } \\
\hline Intervals of $A R A$ & $\begin{array}{l}\text { Pattern } \\
\left(l_{1}^{j_{1}}, l_{2}^{j_{2}}, l_{3}^{j_{3}}, l_{4}^{j_{4}}\right)\end{array}$ & $\begin{array}{l}\text { Index } \\
\text { CARA }\end{array}$ & Tr. 1 & Tr. 2 & Tr. 3 & All & $\%$ TOT \\
\hline $0.077<A R A<+\infty$ & $(A, A, A, A)$ & 9 & 1 & 3 & 0 & 4 & 7.41 \\
\hline $0.054<A R A<0.077$ & $(B, A, B, A)$ & 8 & 0 & 3 & 2 & 5 & 9.26 \\
\hline $0.046<A R A<0.054$ & $(B, B, B, B)$ & 7 & 2 & 3 & 4 & 9 & 16.67 \\
\hline $0.033<A R A<0.046$ & $(C, B, C, B)$ & 6 & 1 & 3 & 1 & 5 & 9.26 \\
\hline $0.032<A R A<0.033$ & $(D, B, D, B)$ & 5 & 2 & 0 & 1 & 3 & 5.56 \\
\hline $0.027<A R A<0.032$ & $(D, C, D, B)$ & 4 & 0 & 2 & 0 & 2 & 3.70 \\
\hline $0.023<A R A<0.027$ & $(D, C, D, C)$ & 3 & 2 & 0 & 1 & 3 & 5.56 \\
\hline $0.016<A R A<0.023$ & $(D, D, D, C)$ & 2 & 7 & 1 & 2 & 10 & 18.52 \\
\hline$-\infty<A R A<0.016$ & $(D, D, D, D)$ & 1 & 3 & 6 & 4 & 13 & 24.07 \\
\hline No. of observations & & & 18 & 21 & 15 & 54 & \\
\hline$\%$ Explained & & & 51 & 60 & 43 & 51 & \\
\hline
\end{tabular}

utility functions that can be ordered by a single risk aversion parameter, this allows to compute for each task three critical degrees of risk aversion, one for indifference between the least risky lottery $A$ and the riskier lottery $B$, one for indifference between lotteries $B$ and $C$, and one for indifference between lotteries $C$ and $D$.

Suppose first that the subject has Constant Absolute Risk Aversion (henceforth $C A R A)$, so that $u(c)=1-\exp (-A R A c)$ for all $c$. Under this specification, one can compute for task 1 the critical $A R A_{1}^{A B}$ that yields indifference between lotteries $A$ and $B$ :

$$
\frac{1}{2} \exp \left(-A R A_{1}^{A B} \bar{x}_{1}^{A}\right)+\frac{1}{2} \exp \left(-A R A_{1}^{A B} \underline{x}_{1}^{A}\right)=\frac{1}{2} \exp \left(-A R A_{1}^{A B} \bar{x}_{1}^{B}\right)+\frac{1}{2} \exp \left(-A R A_{1}^{A B} \underline{x}_{1}^{B}\right)
$$

From the above formula, $A R A_{1}^{A B}=0.077$. A similar method can be used for the other pairs of lotteries $(B, C)$ and $(C, D)$, and for the other tasks 2, 3, and 4. Under $C A R A$, it is well known (e.g., Gollier 2001) that the optimal portfolio composition is independent of initial wealth. From Table 3, it can be shown that tasks 1 and 3 correspond to the same portfolio problem, but with different initial wealth levels, respectively, equal to $w_{1}=8$ and $w_{3}=16$. This implies that $A R A_{1}^{j, j+1}=A R A_{3}^{j, j+1}$ for all pairs of lotteries $(j, j+1)$. In other words, a $C A R A$ subject should answer in exactly the same way for these two tasks. A similar o4bservation can be made for tasks 2 and 4 (see Table 4). The interpretation of Table 4 is the following: if the subject's $A R A$ is inside the interval $(0.054,0.077)$, then she should pick the pattern $\left(l_{1}^{j_{1}}, l_{2}^{j_{2}}, l_{3}^{j_{3}}, l_{4}^{j_{4}}\right)=(B, A, B, A)$ in the four portfolio choice problems, and here, $C A R A$ index is 8 . Notice that the higher the subject's degree of risk aversion, the higher her CARA index, the less risky is the pattern she chooses. Table 4 shows that in our experiment, more than $1 / 2$ of subjects select lotteries in tasks 1-4 in a way 
that is compatible with $C A R A .{ }^{10}$ Results of the elicitation are provided disentangled by treatments in order to show possible differences in the distribution of the CARA ordering among the three subject pools. Indeed, although the percentage of explained patterns is higher for subjects participating in treatment 2, no significant difference is found in the distribution of CARA ordering in the three treatments (see Sect. 4.1).

Now suppose that the subject has Constant Relative Risk $A$ verse (henceforth $C R R A$ ), so that $u(c)=c^{1-R R A} /(1-R R A)$ for all $c$. Under this specification, one can compute for task 1 the critical $R R A_{1}^{A B}$ that yields indifference between lotteries $A$ and $B$ :

$$
\frac{1}{2}\left(\bar{x}_{1}^{A}\right)^{1-R R A_{1}^{A B}}+\frac{1}{2}\left(\underline{x}_{1}^{A}\right)^{1-R R A_{1}^{A B}}=\frac{1}{2}\left(\bar{x}_{1}^{B}\right)^{1-R R A_{1}^{A B}}+\frac{1}{2}\left(\underline{x}_{1}^{B}\right)^{1-R R A_{1}^{A B}}
$$

From the above formula, $R R A_{1}^{A B}=1.320$. A similar method can be used for the other pairs of lotteries $(B, C)$ and $(C, D)$, and for the other tasks 2, 3, and 4. In Table $5 C R R A$ subjects are ordered according to their lottery choices in tasks 1-4. The interpretation of Table 5 is the same as in Table 4, with RRA in place of ARA. Again, the higher the subject's degree of risk aversion, the higher her CRRA index, the less risky is the pattern she chooses. Table 5 shows that, in our experiment, almost 3/4 of subjects have a quadruplet of choices that is compatible with $C R R A .{ }^{11}$ Although the percentage of explained patterns is lower for subjects participating in treatment 2, no significant difference is found in the distribution of CRRA ordering in the three treatments (see Sect. 4.1).

Tasks 1-4 have been designed such that both a CARA subject and a CRRA subject, in order to show that she is not risk-averse (respectively, $R R A \leq 0$ and $A R A \leq 0$ ), should pick the riskiest pattern $\left(l_{1}^{j_{1}}, l_{2}^{j_{2}}, l_{3}^{j_{3}}, l_{4}^{j_{4}}\right)=(D, D, D, D)$, thereby being assigned (CARA or CRRA) index 1. That is why, independently from the assumption of CARA or $C R R A$, if the number of explained patterns is the same under the two specifications, by construction the same percentage of non-risk-averse subjects should be seen. Indeed, we find that this percentage is the same under the two specifications, although $C R R A$ captures a higher number of patterns than $C A R A$ : around $1 / 4$ of the explained patterns are compatible with risk neutrality or risk proneness. This percentage is close to the one found in other experimental studies on risk-attitude elicitation in simple lotteries. Indeed, the whole distribution of $R R A$ in Table 5 is very close to those in the real-payoff tasks (Table 3, p. 1649) of Holt and Laury (2002) and of follow-up studies. ${ }^{12}$

\footnotetext{
10 When checking if a behavioral pattern in tasks $1-4$ is compatible with $C A R A$, we allow up to only one possible deviation of at most one lottery $l_{t}^{j_{t}}$ from each of the theoretical patterns. For example, we assign a $C A R A$ index to pattern $(B, C, B, B)$, namely index 7 , but we assign no index to $(B, D, B, B)$ or to $(C, C, B, B)$.

11 As for Table 4, when checking if a behavioral pattern in tasks 1-4 is compatible with CARA, we allow up to only one possible deviation of at most one lottery $l_{t}^{j_{t}}$ from each of the theoretical patterns. For example, we assign a $C R R A$ index to pattern $(B, B, C, B)$, namely index 8 , but we assign no index to $(B, C, C, B)$ or to $(C, B, C, B)$.

12 Tasks 1-4 contain lotteries whose expected payoffs are between the expected (real) payoff of lotteries $1 \mathrm{X}$ and 20X in Holt and Laury (2002). Although RRA intervals are not perfectly coincident between Table 5 in this paper and their Table 3, the similarity of results is impressive: our study finds $5.26 \%$ of subjects with $R R A \in(1.320,+\infty)$, and they find $1 \%$ and $6 \%$ of subjects with $R R A \in(1.370,+\infty)$,
} 
Table 5 Optimal answers for tasks 1-4 under CRRA

\begin{tabular}{|c|c|c|c|c|c|c|c|}
\hline \multicolumn{3}{|c|}{ Predicted pattern under $C R R A$} & \multicolumn{5}{|c|}{ Experimental Data } \\
\hline Intervals of $R R A$ & $\begin{array}{l}\text { Pattern } \\
\left(l_{1}^{j_{1}}, l_{2}^{j_{2}}, l_{3}^{j_{3}}, l_{4}^{j_{4}}\right)\end{array}$ & $\begin{array}{l}\text { Index } \\
C R R A\end{array}$ & Tr. 1 & Tr. 2 & Tr. 3 & All & $\%$ TOT \\
\hline $1.320<R R A<+\infty$ & $(A, A, A, A)$ & 12 & 1 & 3 & 0 & 4 & 5.26 \\
\hline $0.890<R R A<1.320$ & $(A, A, B, A)$ & 11 & 1 & 0 & 3 & 4 & 5.26 \\
\hline $0.805<R R A<0.890$ & $(A, A, B, B)$ & 10 & 1 & 1 & 2 & 4 & 5.26 \\
\hline $0.670<R R A<0.805$ & $(A, A, C, B)$ & 9 & 0 & 0 & 1 & 1 & 1.32 \\
\hline $0.575<R R A<0.670$ & $(B, A, C, B)$ & 8 & 3 & 5 & 4 & 12 & 15.79 \\
\hline $0.440<R R A<0.575$ & $(B, A, D, B)$ & 7 & 3 & 2 & 2 & 7 & 9.21 \\
\hline $0.439<R R A<0.440$ & $(B, A, D, C)$ & 6 & 0 & 0 & 2 & 2 & 2.63 \\
\hline $0.382<R R A<0.439$ & $(B, B, D, C)$ & 5 & 5 & 2 & 3 & 10 & 13.16 \\
\hline $0.244<R R A<0.382$ & $(C, B, D, C)$ & 4 & 3 & 1 & 2 & 6 & 7.89 \\
\hline $0.197<R R A<0.244$ & $(C, C, D, D)$ & 3 & 2 & 1 & 2 & 5 & 6.58 \\
\hline $0.123<R R A<0.197$ & $(D, C, D, D)$ & 2 & 0 & 0 & 1 & 1 & 1.32 \\
\hline$-\infty<R R A<0.123$ & $(D, D, D, D)$ & 1 & 9 & 6 & 5 & 20 & 26.32 \\
\hline No. of observations & & & 28 & 21 & 27 & 76 & \\
\hline$\%$ Explained & & & 80 & 60 & 77 & 72 & \\
\hline
\end{tabular}

Through the $B D M$ mechanism proposed in task 5, a risk-averse (-loving) subject should declare a certainty equivalent for $l_{4}^{j_{4}}$ - the simple lottery she has been assigned in task 5-lower (higher) than its expected value, i.e.,

$$
C E\left(l_{4}^{j_{4}}\right)<(>) E V\left(l_{4}^{j_{4}}\right) .
$$

Given that in task 5 the lottery assigned to the subject is the same she has chosen in task $4, l_{4}^{j_{4}}$, the proposed portfolio choice problem provides a theoretical prediction on $C E\left(l_{4}^{j_{4}}\right)$ in task 5 both under $C A R A$ and under $C R R A$ specification. Suppose that the subject's pattern in tasks $1-4$ is compatible with $C A R A$. Then, given her $C A R A$ index $h=1,2, \ldots, 9$, her $A R A$ belongs to the interval $\left(\underline{A R A}_{h}, \overline{A R A}_{h}\right)$ for each $h$. Hence, given $l_{4}^{j_{4}}=\left(\bar{x}_{4}^{j_{4}}, 0.5 ; \underline{x}_{4}^{j_{4}}, 0.5\right), \underline{A R A}_{h}$, and $\overline{A R A}_{h}$, it is

\section{Footnote 12 continued}

respectively, in the " $1 \mathrm{X}$ real" and the "20X real" payoffs task (stay in bed); our study finds $5.26 \%$ of subjects with $R R A \in(0.890,1.320)$, and they find $3 \%$ and $11 \%$ of subjects with $R R A \in(0.970,1.370)$, respectively, in "1X real" and "20X real" (highly-risk-averse); our study finds $63.16 \%$ of subjects with $R R A \in(0.123,0.890)$, and they find $62 \%$ and $64 \%$ of subjects with $R R A \in(0.150,0.970)$, respectively, in " $1 \mathrm{X}$ real" and " $20 \mathrm{X}$ real" (from very-risk-averse to slightly-risk-averse); our study finds $26.32 \%$ of subjects with $R R A \in(-\infty, 0.123)$, and they find $34 \%$ and $19 \%$ of subjects with $R R A \in(-\infty, 0.150)$, respectively, in " $1 \mathrm{X}$ real" and "20X real" (from risk-neutral to highly-risk-loving). Notice also that the distribution of $R R A$ in Table 5 in this paper is not very different from $1 \mathrm{X}$ and $20 \mathrm{X}$ real-payoff single unordered tasks in Holt and Laury (2005) and from $1 \mathrm{X}$ and 10X real-payoff single unordered tasks in Harrison et al. (2005). For example, the percentage of non-risk-averse subjects (from risk-neutral to highly-risk-loving) under CRRA in tasks $1-4$ of our experiment (23.32\%) is between those found by Harrison et al. (2005) in 1X and 10X real-payoff single unordered tasks ( $31.71 \%$ and $12.73 \%$, respectively). 


$$
C E\left(l_{4}^{j_{4}} ; A R A_{h}\right)=-\frac{1}{A R A_{h}} \ln \left(\frac{1}{2} \exp \left(-A R A_{h} \bar{x}_{4}^{j_{4}}\right)+\frac{1}{2} \exp \left(-A R A_{h} \underline{x}_{4}^{j_{4}}\right)\right)
$$

with $A R A_{h}=\underline{A R A}_{h}, \overline{A R A}_{h}$. Then, it should be $C E\left(l_{4}^{j_{4}}\right) \in\left(C E\left(l_{4}^{j_{4}} ; \overline{A R A}_{h}\right)\right.$, $\left.C E\left(l_{4}^{j_{4}} ; \underline{A R A}_{h}\right)\right)$. If the subject's pattern in tasks $1-4$ is compatible with $C R R A$, then, given $l_{4}^{j_{4}}$ and her $C R R A$ index $k=1,2, \ldots, 12$, her $R R A$ belongs to the interval $\left(\underline{R R A}_{k}, \overline{R R A}_{k}\right)$ for each $k$. Hence, given $l_{4}^{j_{4}}=\left(\bar{x}_{4}^{j_{4}}, 0.5 ; \underline{x}_{4}^{j_{4}}, 0.5\right), \underline{R R A_{k}}$, and $\overline{R R A}_{k}$, it is

$$
C E\left(l_{4}^{j_{4}} ; R R A_{k}\right)=\left(\frac{1}{2}\left(\bar{x}_{4}^{j_{4}}\right)^{1-R R A}+\frac{1}{2}\left(\underline{x}_{4}^{j_{4}}\right)^{1-R R A}\right)^{\frac{1}{1-R R A}}
$$

with $R R A_{k}=\underline{R R A}_{k}, \overline{R R A}_{k}$. Then, it should be $C E\left(l_{4}^{j_{4}}\right) \in\left(C E\left(l_{4}^{j_{4}} ; \underline{R R A}_{k}\right)\right.$, $\left.C E\left(l_{4}^{j} ; \overline{R R A}_{k}\right)\right)$.

The subject's optimal behavior in tasks 6-10 will be now analyzed.

Consider a two-stage lottery $L$ where the second stage is represented by a set of $n+1$ lotteries $\widetilde{l}_{\theta} \sim\left(x_{1}, p_{1 \theta} ; \ldots ; x_{S}, p_{S \theta}\right)$, with possible payoffs $x_{1}>\cdots>x_{S}$, $\theta \in\{0, \ldots, n\}, p_{s \theta} \geq 0$ and $\Sigma_{s=1}^{S} p_{s \theta}=1$. The first stage is represented by the lottery $L$ having as possible outcomes the second-stage lotteries $\widetilde{l}_{\theta}$ with probabilities $\left(q_{0}, \ldots, q_{n}\right)$, with $q_{\theta} \geq 0$ and $\Sigma_{\theta=0}^{n} q_{\theta}=1$. These are the second-order probabilities over the plausible probability distributions for $\widetilde{l}_{\theta}$.

In all treatments of our experiment, the impact of information on the value of lotteries is modeled in the $K M M$ framework. Following $K M M$, it is assumed that the subject's ex ante utility is measured by:

$$
u(C E(L))=\phi^{-1}\left(\sum_{\theta=0}^{n} q_{\theta} \phi\left(E U\left(\widetilde{l}_{\theta}\right)\right)\right)
$$

with

$$
E U\left(\widetilde{l}_{\theta}\right)=\sum_{s=1}^{S} p_{s \theta} u\left(x_{s}\right)
$$

Function $u$ is a von Neumann-Morgenstern utility function, and $\phi$ captures the subject's smooth ambiguity attitude. In fact, $\phi$ is a von Neumann-Morgenstern index function accounting for the attitude toward mean-preserving spreads in the induced distribution of the expected utility of the one-stage lottery conditional to $\theta$, namely $E U\left(\widetilde{l}_{\theta}\right) . K M M$ define "smooth ambiguity aversion" and show that it is equivalent to $\phi$ being concave. Therefore, it is equivalent to aversion to mean-preserving spreads of the expected utility values induced by the second-order subjective probability and lottery $\widetilde{l}_{\theta}$. Then, defining function $v$ as $v=\phi \circ u$, the certainty equivalent of the two-stage lottery is

$$
C E(L)=v^{-1}\left(\sum_{\theta=0}^{n} q_{\theta} v\left(C E\left(\widetilde{l}_{\theta}\right)\right)\right)
$$


where $C E\left(\widetilde{l}_{\theta}\right)$ is the certainty equivalent of the one-stage lottery conditional to $\theta$. Function $v$ is a von Neumann-Morgenstern index function accounting for the attitude toward mean-preserving spreads in certainty equivalents of the one-stage lottery conditional to $\theta$, namely $C E\left(\widetilde{l}_{\theta}\right)$.

In each task of our experiment, there are only two possible payoffs, namely $\bar{x}, \underline{x} \in$ $\mathbb{R}_{+}, \bar{x}>\underline{x}$. Therefore, the small urn is represented by the 10 -ball one-stage lottery $\widetilde{l}_{\theta} \sim\left(\bar{x}, \bar{p}_{\theta} ; \underline{x}, 1-p_{\theta}\right)$, where $p_{\theta}=\frac{\theta}{10}$ is the objective probability given by the ratio of the number of white balls $\theta \in\{0,1, \ldots, 10\}$ over 10 . The second-order probabilities on the possible compositions of the small urn depend upon the treatment under consideration. In tasks 6-10 of treatments 1 and 2, the probability distribution $\left(q_{0}, \ldots, q_{10}\right)$ over the one-stage lotteries is objective. It is binomial in treatment 1 and uniform in treatment 2 . Therefore, in treatment 1 , given a task from 6 to 10 , the secondorder objective probabilities are always less dispersed than in the corresponding task in treatment $2 .{ }^{13}$

Since in our experiment second-stage lotteries assigned to a subject in tasks 5-9 have the same pair of outcomes, their variety only depends on first-order probabilities. Notice that the simple lottery in task $5, l_{4}^{j_{4}}$, is analogous to a two-stage lottery with all second-stage lotteries $\widetilde{l}_{\theta=5}$ being $l_{4}^{j_{4}}$, namely $L_{5}:=\left(q_{1}, l_{4}^{j_{4}} ; q_{2}, l_{4}^{j_{4}} ; . . ; q_{n}, l_{4}^{j_{4}}\right)$. It is trivially assumed that $L_{5} \sim l_{4}^{j_{4}}$. In order to identify whether a subject shows aversion, neutrality, or proneness to ambiguity, the subject's answers to tasks 5 and 6 can be compared. In task 5, the subject is asked to value the unambiguous lottery that she has selected in task 4. In task 6, the subject is asked to do the same for an ambiguous urn with the same expected probability for the two outcomes. This suggests the following operational Definition 1.

Definition 1 (value-ambiguity attitude) Call $C E\left(L_{t}\right)$ the subject's reservation price for the two-stage lottery assigned in task $t \in\{5,6\}$. It can be interpreted as the certainty equivalent of the two-stage lottery in task $t$. Then, a subject is value-ambiguity-averse if $C E\left(L_{6}\right) \leq C E\left(L_{5}\right)$. She is value-ambiguity-neutral if $C E\left(L_{6}\right)=C E\left(L_{5}\right)$. She is value-ambiguity-loving if $C E\left(L_{6}\right) \geq C E\left(L_{5}\right)$.

In short, a value-ambiguity-averse subject values an ambiguous lottery less than its unambiguous equivalent with the same mean probabilities. In the $K M M$ model, this is true if the subject's $\phi$ function is concave.

Our experimental design offers an alternative to elicit ambiguity aversion by comparing the subject's answers to tasks 4 and 10. The two possible outcomes in lotteries $\{A, B, C, D\}$ are the same in the two tasks. The difference lies in the fact that probabili-

\footnotetext{
13 In particular, in treatment 1 , the objective second-order probabilities are as follows: in tasks 6 and $10, q_{10}=q_{0}=1 / 1024 \simeq 0.1 \%, q_{9}=q_{1}=10 / 1024 \simeq 1 \%, q_{8}=q_{2}=45 / 1024 \simeq 4.4 \%$, $q_{7}=q_{3}=120 / 1024 \simeq 11.7 \%, q_{6}=q_{4}=210 / 1024 \simeq 20.5 \%$, and $q_{5}=252 / 1024 \simeq 24.6 \%$; in task $7, q_{7}=q_{3}=1 / 16=6.25 \%, q_{6}=q_{4}=4 / 16=25 \%$, and $q_{5}=6 / 16=37.5 \%$; in task $8, q_{10}=q_{3}=1 / 128 \simeq 0.8 \%, q_{9}=q_{4}=7 / 128 \simeq 5.5 \%, q_{8}=q_{5}=21 / 128 \simeq 16.4 \%$, $q_{7}=q_{6}=35 / 128 \simeq 27.3 \% ;$ in task $9, q_{7}=q_{0}=1 / 128 \simeq 0.8 \%, q_{6}=q_{1}=7 / 128 \simeq 5.5 \%$, $q_{5}=q_{2}=21 / 128 \simeq 16.4 \%, q_{4}=q_{3}=35 / 128 \simeq 27.3 \%$. All other $q_{\theta}$ are zero. In treatment 2 , the objective second-order probabilities are: in tasks 6 and $10, q_{\theta}=1 / 11 \simeq 9.1 \%$ for every $\theta=0,1, \ldots, 10$; in task $7, q_{\theta}=1 / 5$ for every $\theta=3,4, \ldots, 7$; in task $8, q_{\theta}=1 / 8$ for every $\theta=3,4, \ldots, 10$; in task 9 , $q_{\theta}=1 / 8$ for every $\theta=0,1, \ldots, 7$. All other $q_{\theta}$ are zero.
} 
ties are unambiguously $1 / 2$ in task 4 , whereas they are ambiguous in task 10 , with mean 1/2. Defining a dispersion order $\succ$ on set $\{A, B, C, D\}$, such that $D \succ C \succ B \succ A$, a more dispersed lottery is equivalent to a portfolio containing a larger share invested in the risky asset.

Definition 2 (choice-ambiguity attitude) Call $j_{t} \in\{A, B, C, D\}$ the index of the lottery chosen by a subject in task $t \in\{4,10\}$. Then, a subject is choice-ambiguityaverse if $j_{10} \preceq j_{4}$, i.e., if lottery $j_{10}$ is not more dispersed than lottery $j_{4}$. She is choice-ambiguity-neutral if $j_{10}=j_{4}$. She is choice-ambiguity-loving if $j_{10} \succeq j_{4}$, i.e., lottery $j_{10}$ is not less dispersed than $j_{4}$.

Equivalently, a choice-ambiguity-averse subject will always reduce her demand for the risky asset when the distribution of outcomes becomes ambiguous. In the $K M M$ smooth ambiguity aversion framework, Gollier (2012) has shown that it is not true in general that the concavity of the $\phi$ function implies the subject's choiceambiguity aversion. In other words, a smooth ambiguity-averse subject could have a larger demand for the ambiguous asset than another ambiguity-neutral subject with the same risk aversion. However, Gollier (2012) provides a set of sufficient conditions on the structure of the two-stage uncertainty to re-establish the link between the concavity of $\phi$ and ambiguity aversion. One of these sufficient conditions is that the different second-stage distributions of the risky asset can be ordered by the Monotone Likelihood Ratio stochastic order. Referring to the risky assets in Table 3, the set of distributions of returns $\left\{\left(\bar{y}_{t}, p_{\theta} ; \underline{y}_{t}, 1-p_{\theta}\right) \mid \theta=0, \ldots, 10\right\}$ in our ambiguous tasks can always be ordered by the Monotone Likelihood Ratio. Thus, we conclude that, in the $K M M$ framework, the two definitions of value-ambiguity aversion and choiceambiguity aversion are equivalent in our experimental setting and are satisfied if $\phi$ is concave. This justifies the following definition.

Definition 3 (coherent-ambiguity attitude) A subject is coherently-ambiguity-averse if $C E\left(L_{6}\right) \leq C E\left(L_{5}\right)$ and $j_{10} \preceq j_{4}$, with at least one of the two relations holding strictly. She is coherently-ambiguity-neutral if $C E\left(L_{6}\right)=C E\left(L_{5}\right)$ and $j_{10}=j_{4}$. She is coherently-ambiguity-loving if $C E\left(L_{6}\right) \geq C E\left(L_{5}\right)$ and $j_{10} \succeq j_{4}$, with at least one of the two inequalities holding strictly.

Our operational definition of coherent-ambiguity attitude is based on a doublecheck: the subject's behavior is compared in task 5 versus task 6 and in task 4 versus task 10. The first comparison shows whether, given the two second-stage lottery outcomes, the subject prefers to know first-order probability $p_{\theta}$ than facing a mean-preserving spread of second-order probabilities over the all possible $p_{\theta}$. The second comparison shows whether the subject prefers a less risky lottery (a less dispersed performance of the portfolio in Table 3) where this mean-preserving spread takes place.

The analysis will now turn to analyze how the certainty equivalent of the twostage lottery varies when moving from task 6 to tasks 7,8 , or 9 and whether this variation depends on the fact that the subject is ambiguity-averse. First $C E\left(L_{7}\right)$ will be compared with $C E\left(L_{6}\right)$. It should be remembered that, in each of our three treatments, the two-stage lottery in task 7 is obtained from task 6 by symmetrically eliminating the plausibility of the extreme urns $\theta=0,1,2,8,9,10$. This necessarily implies 
$q_{\theta}=0$ in task 7 for these $\theta$. Compared to task 6 , the subject's subjective secondorder probabilities must be symmetrically transferred from the extreme urns to the less dispersed urns $\theta=3, \ldots, 7$. This yields a mean-preserving contraction in the distribution of $\widetilde{U} \sim\left(E U\left(\widetilde{l}_{0}\right), q_{0} ; \ldots ; E U\left(\widetilde{l}_{10}\right), q_{10}\right)$, as will be shown. In the remainder of this section, $u$ is normalized in such a way that $u\left(x_{4}^{j_{4}}\right)=0$ and $u\left(\bar{x}_{4}^{j_{4}}\right)=1$, so that $E U\left(\widetilde{l_{\theta}}\right)=p_{\theta}$.

Lemma 4 Consider a symmetric random variable $\widetilde{p} \sim\left(p_{0}, q_{0} ; \ldots ; p_{n}, q_{n}\right)$, with $p_{\theta}=\theta / n, q_{\theta}=q_{n-\theta}$ for all $\theta$, and $n>2$. Consider another symmetric random variable $\tilde{p}^{\prime} \sim\left(p_{0}, q_{0}^{\prime} ; \ldots ; p_{n}, q_{n}^{\prime}\right)$ on the same support, but with $q_{0}^{\prime}=q_{n}^{\prime}=0$ and $q_{\theta}^{\prime}=q_{n-\theta}^{\prime} \geq q_{\theta}=q_{n-\theta}$ for all $\theta \in\{1, \ldots, n-1\}$. It implies that $E \phi\left(\tilde{p}^{\prime}\right) \geq$ $E \phi(\widetilde{p})$ for all concave functions $\phi$, i.e., that $\widetilde{p}^{\prime}$ is a Rothschild-Stiglitz mean-preserving contraction of $\widetilde{p}$.

Proof Observe that, by symmetry,

$$
E \widetilde{p}=\sum_{\theta=0}^{n} q_{\theta} \frac{\theta}{n}=\sum_{\theta=0}^{n / 2} q_{\theta}\left(\frac{\theta}{n}+\frac{n-\theta}{n}\right)=\sum_{\theta=0}^{n / 2} q_{\theta}=\frac{1}{2}
$$

Because the same observation can be made for $\widetilde{p}^{\prime}, E \widetilde{p}=E \widetilde{p}^{\prime}=1 / 2$. Because $\widetilde{p}^{\prime}$ is obtained from $\widetilde{p}$ by a transfer of probability mass from the extreme states to the center of the distribution, it is concluded that $\widetilde{p}$ is a mean-preserving spread of $\widetilde{p}^{\prime}$.

Repeating this lemma three times, it turns out that $C E\left(L_{7}\right)$ must be larger than $C E\left(L_{6}\right)$ under smooth ambiguity aversion. Because $L_{7}$ is still ambiguous, $C E\left(L_{7}\right)$ is smaller than $C E\left(L_{5}\right)$. Thus, $C E\left(L_{5}\right) \geq C E\left(L_{7}\right) \geq C E\left(L_{6}\right)$. The opposite result would hold under smooth ambiguity proneness. Observe that a crucial assumption for the lemma is the symmetry of the second-order probability distributions. In treatments 1 and 2, the second-order probability distribution on the composition of the small urn is either binomial or uniform: both are clearly symmetric. In treatment 3 , the symmetry of the second-order probability distribution will depend upon the subject's beliefs on the composition of the big urn from which the small urn is built. However, the principle of insufficient reason suggests that the subject has symmetric beliefs on the composition of the big urn, and, therefore, on the composition of the small urn generated by the Bernoullian process. Under this principle, the following proposition can be written.

Proposition 5 If the subject is ambiguity-averse, then $C E\left(L_{5}\right) \geq C E\left(L_{7}\right) \geq C E\left(L_{6}\right)$. If she is ambiguity-loving, then $C E\left(L_{5}\right) \leq C E\left(L_{7}\right) \leq C E\left(L_{6}\right)$. If she is ambiguityneutral, then $C E\left(L_{5}\right)=C E\left(L_{7}\right)=C E\left(L_{6}\right)$.

Tasks 8 and 9 will now be compared to task 6 . Task 8 is similar to task 6 except that the worst urns have been eliminated. Proposition 6 shows that the certainty equivalent of the two-stage lottery assigned in task 8 is greater than the one of the two-stage lottery assigned in task 6, whatever the degree of ambiguity of the subject, hence independently of the fact that she is ambiguity-averse, neutral, or loving. The opposite result prevails for task 9 , in which the best urns have been removed. Therefore, comparison among tasks 6,8 , and 9 always leads to $C E\left(L_{8}\right) \geq C E\left(L_{6}\right) \geq C E\left(L_{9}\right)$, whatever the subject's attitude toward ambiguity. 
Proposition 6 Suppose that new information implies that the worst (best) urns become implausible, without reducing the probability $q_{\theta}$ of any of the other urns. This new information raises (reduces) the certainty equivalent of the two-stage lottery independent of the degree of ambiguity aversion.

Proof Because $\phi$ is increasing and concave, it is obvious that any first-degree or second-degree stochastic dominance improving shift in the distribution of $\left(q_{0}, E U\left(\widetilde{l}_{0}\right) ; \ldots ; q_{n}, E U\left(\widetilde{l}_{n}\right)\right)$ raises the certainty equivalent of the two-stage lottery. Because $p_{\theta}=\theta / n$ is increasing in $\theta$, so is $E U\left(\widetilde{l}_{\theta}\right)=p_{\theta} u\left(\bar{x}_{4}^{j_{4}}\right)+\left(1-p_{\theta}\right) u\left(\underline{x}_{4}^{j_{4}}\right)$. Suppose that new information makes the worst lotteries $\left(\widetilde{l}_{0}, \widetilde{l}_{1}, \ldots, \widetilde{l}_{m}\right), m<n$, totally implausible. This implies that the new second-order probabilities take the form $\left(\widehat{q}_{0}, \widehat{q}_{1}, \ldots, \widehat{q}_{n}\right)$, with $\widehat{q}_{0}=\widehat{q}_{1}=\cdots=\widehat{q}_{m}=0$. This yields a first-degree stochastic improvement if $\widehat{q}_{i} \geq q_{i}$ for all $i \in\{m+1, \ldots, n\}$. Therefore, under the assumption that both $u$ and $\phi$ are strictly monotone, this new information raises the certainty equivalent of the lottery independent of the degree of ambiguity aversion. Of course, the symmetric case also holds. Suppose that new information implies that the best scenarios become implausible, without reducing the probability $q_{\theta}$ of any of the other scenarios. This new information reduces the certainty equivalent of the lottery independent of the degree of ambiguity aversion.

This result also applies to the comparison between task 8 (task 9) and task 7: the certainty equivalent of the two-stage lottery assigned in the former task must be greater (smaller) than the one of the two-stage lottery assigned in task 6, whatever the degree of ambiguity of the subject. Task 7 may be seen as a modification of task 9 through new information implying that the worst scenarios become implausible, without reducing the probability $q_{\theta}$ of any of the other scenarios in task 9 . Task 7 may be also seen as a modification of Task 8 through new information implying that the best scenarios become implausible, without reducing the probability $q_{\theta}$ of any of the other scenarios in task 8. Therefore, it follows that $C E\left(L_{8}\right) \geq C E\left(L_{7}\right) \geq C E\left(L_{9}\right)$ independent of the shape of $\phi$.

An attempt will now be made to establish the complete ranking of the values of tasks 5-9 under smooth ambiguity aversion. Earlier, it has been noted that smooth ambiguity aversion implies that $C E\left(L_{5}\right) \geq C E\left(L_{7}\right) \geq C E\left(L_{6}\right)$. Combining these three sequences of inequalities implies that, under smooth ambiguity aversion,

$$
\left.\begin{array}{l}
C E\left(L_{5}\right) \\
C E\left(L_{8}\right)
\end{array}\right\} \geq C E\left(L_{7}\right) \geq C E\left(L_{6}\right) \geq C E\left(L_{9}\right)
$$

independent of the subject's attitude toward risk. The only degree of freedom under smooth ambiguity aversion is thus given by the relative values of task 5 (no ambiguity: $q_{5}=1$ ) and task 8 (ambiguity with worst urns eliminated: $q_{0}=q_{1}=q_{2}=0$ ). If ambiguity aversion is small enough, i.e., the concavity of $\phi$ is small, then the large expected probability of the high outcome enjoyed in task 8 will dominate the ambiguity aversion effect to yield $C E\left(L_{8}\right)>C E\left(L_{5}\right)$, otherwise $C E\left(L_{8}\right) \leq C E\left(L_{5}\right)$. The following result is a direct consequence of Gollier (2001, Sect. 6.3.2). 
Proposition 7 Suppose that a subject prefers the unambiguous lottery $L_{5}$ to the ambiguous lottery $L_{8}$ (this is possible only under ambiguity aversion). Then, an increase in ambiguity aversion in the KMM model can never reverse this ranking.

This implies that, assuming similar attitudes toward risk, any subject with $C E\left(L_{5}\right)<$ $C E\left(L_{8}\right)$ has a smaller degree of smooth ambiguity aversion than any subject with $C E\left(L_{5}\right) \geq C E\left(L_{8}\right)$. Thus, comparing the values of task 5 and 8 for an ambiguityaverse subject will provide information about her degree of ambiguity aversion.

Of course, in the limit case of smooth ambiguity-neutrality,

$$
C E\left(L_{8}\right)>C E\left(L_{5}\right)=C E\left(L_{6}\right)=C E\left(L_{7}\right)>C E\left(L_{9}\right),
$$

independent of the subject's attitude toward risk. Finally, for an ambiguity-loving subject,

$$
C E\left(L_{8}\right) \geq C E\left(L_{6}\right) \geq C E\left(L_{7}\right) \geq\left\{\begin{array}{l}
C E\left(L_{5}\right) \\
C E\left(L_{9}\right)
\end{array},\right.
$$

independent of her attitude toward risk. If the degree of ambiguity proneness is small enough, i.e., the convexity of $\phi$ is small, then the low expected probability of the high outcome faced in task 9 will dominate the attractiveness of this ambiguous lottery for ambiguity-loving subjects, so that $C E\left(L_{9}\right)<C E\left(L_{5}\right)$, otherwise $C E\left(L_{9}\right) \geq C E\left(L_{5}\right)$.

Proposition 8 Suppose that a subject prefers the unambiguous lottery $L_{5}$ to the ambiguous lottery $L_{9}$ (this is possible also under ambiguity proneness). Then, a concave transformation of the $\phi$ function in the KMM model can never reverse this ranking.

Thus, comparing the values of task 5 and 9 for an ambiguity-loving subject will provide information about her degree of ambiguity proneness.

The next corollary shows the difference among certainty equivalents of two-stage lotteries in the same task of different treatments. The comparison of treatments 1 and 2 is the easiest. The uniform distribution of the second-order probabilities in treatment 2 is clearly a mean-preserving spread of the binomial distribution obtained in treatment 1. Comparing the certainty equivalents for treatments 1 and 3 is more difficult. In both treatments, a Bernoullian process is applied to build the small urn, but the parameter of the Bernoulli distribution is $p=1 / 2$ in treatment 1 , whereas it is unknown in treatment 3. If one accepts the principle of insufficient reason, then it may be assumed that the third-order probabilities on parameter $p$ yields $E p=1 / 2$. Under this assumption, treatment 3 always yields a mean-preserving spread of the second-order probability distribution $\left(q_{0}, \ldots, q_{n}\right)$. Under ambiguity aversion, this yields a reduction of the certainty equivalents. This yields the following result.

Corollary 9 If the subject is ambiguity-averse (-loving), then $C E_{t}$ is greater (smaller) in treatment 1 than in treatments 2 and 3 for every $t=6,7,8,9$.

By combining Proposition 7, Proposition 8, and Corollary 9, specific behavioral predictions about possible treatment differences can be made. Although our experimental design is not within-subject, Tables 4 and 5 show that the distribution of the degree of risk aversion does not differ among the three treatments, if both a CARA and a 
$C R R A$ specification are used. Hence, if we assume that the distribution of the degree of ambiguity aversion is the same among the three treatments, then for similar degrees of risk aversion it should be found that the percentage of ambiguity-averse subjects with $C E\left(L_{5}\right) \geq C E\left(L_{8}\right)$ is lower in treatment 1 than in treatments 2 and 3 . By combining Proposition 8 and Corollary 9 for ambiguity-loving subjects, a symmetrical prediction arises. Assume that the distribution of the degree of ambiguity proneness is the same among the three treatments. Then, the percentage of ambiguity-loving subjects with $C E\left(L_{9}\right) \geq C E\left(L_{5}\right)$ should be lower in treatment 1 than in treatments 2 and 3 within the same class of risk aversion.

\section{Experimental results}

In this section, our experimental results will be presented. ${ }^{14}$ First, in Sect. 4.1, the results of the elicitation of the subjects' risk attitude through the portfolio choice method implemented in (unambiguous) tasks 1-4 are analyzed. Then, in Sect. 4.2, subjects will be classified according to their ambiguity attitude relying on the operational definition introduced in Sect. 3. In Sect. 4.3, the main theoretical predictions derived in Sect. 3 will be tested. In Sect. 4.4, there will be an analysis of the treatment effects on the distribution of subjects' beliefs over second-order probabilities and on the certainty equivalents of two-stage lotteries in the ambiguous tasks.

\subsection{Risk attitude elicitation}

The portfolio choice method used at the beginning of the experiment enables all subjects to face the same set of lotteries in tasks 1-4. This prompts a risk-attitude ordering of subjects independent of $l_{4}^{j_{4}}$, the lottery chosen in task 4 . This is the first reason why such ordering is preferred to the certainty equivalent elicited in task 5 , which instead depends on $l_{4}^{j_{4}}$. Further, this specific portfolio choice method has the advantage of imposing some theoretically derived constraints which allow to check whether the subject's selected pattern is compatible with a CARA and/or a CRRA specification. This provides an empirical verification of what is generally assumed in many experimental studies on risk aversion.

First, a check is made on whether the portfolio choice method elicitation (tasks 1-4) leads to the same ordering in terms of $C E\left(L_{5}\right)$ as the (more standard) $B D M$ mechanism proposed in task 5 . Indeed, a positive (coeff. $=0.46$ ) and highly significant ( $P$ value $=0.000)$ correlation is found between $C E\left(L_{5}\right)$ as predicted by the CARA ordering derived from the selected pattern in tasks 1-4 (see Table 4 ) and the one elicited through the $B D M$ mechanism in task 5. If the CRRA is used in place of the CARA ordering (see Table 5), the former correlation is slightly lower (coeff. $=0.36)$ and again statistically significant $(P$ value $=0.006) .{ }^{15}$

\footnotetext{
14 All raw data and statistical codes are available on request.

15 More precisely, $C E\left(L_{5}\right)$ predicted by the $C A R A$ ordering is calculated as the average between $C E\left(l_{4}^{j_{4}} ; \overline{A R A}_{h}\right)$ and $C E\left(l_{4}^{j_{4}} ; \underline{A R A}_{h}\right)$ in (1), for $h=1,2, \ldots, 9$. Similarly, $C E\left(L_{5}\right)$ predicted by the $C R R A$ ordering is calculated as the average between $C E\left(l_{4}^{j_{4}} ; \overline{R R A}_{k}\right)$ and $C E\left(l_{4}^{j_{4}} ; \underline{R R A}_{k}\right)$ in $(2)$, for $k=1,2, \ldots, 12$.
} 
Second, significant differences among the three treatments in the distribution of $C A R A$ indexes or in the distribution of CRRA indexes are checked for. Although the percentage of explained patterns under each specification is different in the Uniform treatment (see respectively Tables 4, 5), no significant difference in the distribution of risk-aversion ordering in the three treatments is found. This is what Fig. 8 in the Appendix seems to suggest, if both a CARA and a CRRA specification are used. To provide support to the graphical representation, two non-parametric tests have been conducted: a Kruskal-Wallis test ${ }^{16}$ and a Kolmogorov-Smirnov equality-of-distributions test with a pairwise comparison between treatments ${ }^{17}$. Both tests confirm no significant difference in the distribution of both the CARA and the CRRA ordering among the three treatments. ${ }^{18}$

Therefore, both orderings are correlated with the certainty equivalent of task 5 and lead to similar distributions of risk attitude among treatments. Without assuming whether subjects are $C A R A$ or $C R R A$, both specifications are used when analyzing possible relations between the subject's degree of risk aversion and her behavior in tasks 6-10.

It is true that all the theoretical predictions derived in Sect. 3 within the KMM framework should hold whatever the subject's risk aversion. Nevertheless possible correlations between risk attitude and ambiguity attitude are checked for. A further check is made about a possible role of risk attitude when testing our main theoretical predictions, which have been shown to hold independently of the subject's risk attitude. Finally, including risk aversion as an explanatory variable in the econometric analysis may be useful in order to provide an experimental answer to some open theoretical questions such as the sign of $C E\left(L_{8}\right)-C E\left(L_{5}\right)$ for ambiguity-averse subjects or the sign of $C E\left(L_{9}\right)-C E\left(L_{5}\right)$ for ambiguity-loving ones. Notice that, for ambiguity-neutral subjects, it is always $C E\left(L_{8}\right)>C E\left(L_{5}\right)$ and $C E\left(L_{9}\right)<C E\left(L_{5}\right)$.

\subsection{Ambiguity attitude elicitation}

In Table 6, subjects are classified as being ambiguity-averse, ambiguity-neutral, and ambiguity-loving in each treatment according to Definition 3 (coherent-ambiguity attitude). ${ }^{19}$

\footnotetext{
16 The Kruskal-Wallis equality-of-populations rank test verifies the hypothesis that several samples are from the same population.

17 The Kolmogorov-Smirnov test compares two observed distributions $f(\cdot)$ and $g(\cdot)$. The procedure involves forming the cumulative frequency distributions $F(\cdot)$ and $G(\cdot)$ and finding the size of the largest difference between these. The hypothesis tested is whether the two observed distributions are equal (pairwise comparisons between treatments 1-2, treatments 1-3, and treatments 2-3).

18 According to the Kruskal-Wallis test the null hypothesis of equality of distributions $(P$ value $=0.401$ for $C A R A$ and $P$ value $=0.357$ for $C R R A$ ) cannot be rejected. The Kolmogorov-Smirnov test confirms this result.

$19 E U$ maximizing subjects for the Binomial and Uniform treatments and subjective expected utility (henceforth, $S E U$ ) maximizing subjects for the Unknown are both value-ambiguity-neutral and choice-ambiguityneutral, hence coherent-ambiguity-neutral. However, given that the choice set in all our experimental tasks is discrete, it cannot be excluded that weekly non- $E U$ (and non-SEU) maximizing subjects may fall into the group of coherent-ambiguity-neutral subjects. For example, consider a non-EU maximizing subject with
} 
G. Attanasi et al.

Table 6 Classification of (coherent-)ambiguity attitude according to Definition 3

\begin{tabular}{|c|c|c|c|c|}
\hline & Binomial & Uniform & Unknown & Total \\
\hline Coherent averse & 13 & 15 & 17 & 45 \\
\hline Coherent neutral & 16 & 5 & 8 & 29 \\
\hline Coherent loving & 5 & 9 & 4 & 18 \\
\hline Total classified & 34 & 29 & 29 & 92 \\
\hline $\begin{array}{l}\text { Value averse \& Choice } \\
\text { loving }\end{array}$ & 1 & 4 & 2 & 7 \\
\hline $\begin{array}{l}\text { Value loving \& Choice } \\
\text { averse }\end{array}$ & 0 & 2 & 4 & 6 \\
\hline Total unclassified & 1 & 6 & 6 & 13 \\
\hline
\end{tabular}

Almost $1 / 2$ of the classified subjects are ambiguity-averse, while less than 1/5 are ambiguity-loving. Only 13 subjects (less than $12 \%$ of the sample) participating in our experiment cannot be classified according to Definition 3: around half of them are ambiguity-averse according to Definition 1 (value-ambiguity attitude) and ambiguityloving according to Definition 2 (choice-ambiguity attitude). The other half of them are value-ambiguity-loving and choice-ambiguity-averse. ${ }^{20}$ Given the small percentage of unclassified subjects, it can be concluded that the concavity of the $\phi$ function implies choice-ambiguity aversion in our experimental tasks. This was exactly our theoretical prediction, given that the different second-stage distributions of the risky asset have been set such that they can be ordered according to the Monotone Likelihood Ratio stochastic order (see Gollier 2012). Indeed, the correlation between strong valueambiguity aversion $\left(C E\left(L_{6}\right)<C E\left(L_{5}\right)\right)$ and strong choice-ambiguity aversion $\left(j_{10} \prec\right.$ $\left.j_{4}\right)$ is positive, not very high (coeff. $=0.18$ ), but statistically significant $(P$ value $=0.074$ ). This last result will be further analyzed at the end of Sect. 4.3 , by showing that in our sample subjects with strong choice-ambiguity aversion are usually nonstrongly value-ambiguity-averse.

From Table 6, one can also see that the percentage of classified subjects being ambiguity-averse is lower in the Binomial than in the Uniform treatment and in the Unknown treatment. Further, the percentage of classified subjects being ambiguityneutral is higher in the Binomial than in the other two treatments.

Additional results can be stated by considering the "sign" of the ambiguity attitude. This is defined as being negative if the subject is ambiguity-averse, null if she is ambiguity-neutral, and positive if she is ambiguity-loving. Looking at the multinomial logistic regression of the sign of the ambiguity attitude over the treatment, it is found that the relative risk ratio for being ambiguity-neutral versus being ambiguity-averse is 0.27 ( $P$ value $=0.040)$ when switching from the Binomial to the Uniform treatment and $0.38(P$ value $=0.091)$ when switching from the Binomial to the Unknown

\footnotetext{
Footnote 19 continued

a strictly concave $\phi$ function, hence being ambiguity-averse. If the concavity of her $\phi$ function is small, then in our discrete choice set she could make the same choice as another subject with a linear $\phi$ function, hence ending up being classified as coherent-ambiguity-neutral.

20 Although the number of unclassified subjects is lower in the Binomial than in the other two treatments, unclassified subjects are not statistically different from classified ones both with respect to CARA or CRRA ordering and with respect to the lottery chosen in task 4 .
} 
treatment. In other words, the expected probability of being ambiguity-neutral seems to be higher for subjects who participate in the Binomial treatment. Table 6 also shows that the percentage of subjects being ambiguity-loving is lower in the Binomial than in the Uniform treatment, but not in the Unknown treatment. However, a multinomial logistic regression of the ambiguity attitude over the treatment shows that the relative risk ratio for being ambiguity-loving versus being ambiguity-averse is 1.56 (not statistically significant: $P$ value $=0.510$ ) when switching from the Binomial to the Uniform treatment and 0.62 (not statistically significant: $P$ value $=0.521$ ) when switching from the Binomial to the Unknown treatment. ${ }^{21}$

A possible explanation of this result relies on Corollary 9. Given the degree of ambiguity attitude, $\left|C E\left(L_{6}\right)-C E\left(L_{5}\right)\right|$ is lower in the Binomial than in the Uniform treatment. This is due to the fact that the distribution of second-order probabilities is less dispersed in the Binomial than in the Uniform treatment. Moreover, recall that the set of possible certainty equivalent values that a subject may select is discrete. Therefore, if a subject is slightly-ambiguity-averse or slightly-ambiguity-loving, it is more likely for her to choose $C E\left(L_{6}\right)=C E\left(L_{5}\right)$ in the Binomial than in the Uniform treatment. ${ }^{22}$ The intuition based on Corollary 9 applies also to the comparison between the Binomial and the Unknown treatment. Indeed, the percentage of ambiguity-averse (loving) subjects in the Unknown treatment is higher (lower) with respect to the other two treatments, although this difference is not significant (Kruskal-Wallis, $P$ value $=0.258$ ). However, by making a pairwise comparison between treatments about the percentage of ambiguity-averse subjects, it is found that there is no statistically significant difference between the Binomial and the Uniform treatment ( $t$ test, $P$ value $=0.290)^{23}$ and between the Uniform and the Unknown treatment $(P$ value $=0.605)$, while the difference between the Binomial and the Unknown treatment is almost significant $(P$ value $=0.109)$. This distortion confirms our intuition about the interpretation of the Unknown treatment. In the Binomial and in the Unknown treatment, the 10ball small urn in task 6 has been generated through the same Bernoullian process. However, the latter treatment is intrinsically more ambiguous, given that there is no information about the composition of the big urn from which the small unknown urn is generated. According to $K M M$, this generates smaller $C E\left(L_{6}\right)$ through (4) and/or lower $j_{10}$ through (3) in the Unknown than in the Binomial treatment, thereby significantly increasing the percentage of subjects for which it is $C E\left(L_{6}\right) \leq C E\left(L_{5}\right)$ and $j_{10} \preceq j_{4}$. Notice that if value-ambiguity aversion and choice-ambiguity aversion are disentangled, a higher percentage of ambiguity-averse subjects in the Unknown treatment is found than in the Binomial treatment, although this difference is not statistically significant.

\footnotetext{
21 These results are not shown but can be made available upon request.

22 This intuition is reinforced by the fact that the correlation between (strong) value-ambiguity-aversion and (strong) choice-ambiguity-aversion found above in all the sample of classified subjects is higher (coeff. $=0.45)$ and significant $(P$ value $=0.007)$ only if the analysis is restricted at the Binomial treatment. In this treatment, it is plausible that only highly-ambiguity-averse subjects show at the same time $C E\left(L_{6}\right)<$ $C E\left(L_{5}\right)$ and $j_{10} \prec j_{4}$.

23 The $t$ test is any statistical hypothesis test (parametric) in which the test statistic follows a Student's $\mathrm{t}$ distribution if the null hypothesis is supported. Here, a two-sample $t$ test is run for a difference in mean (the null hypothesis is that the two samples have the same mean).
} 

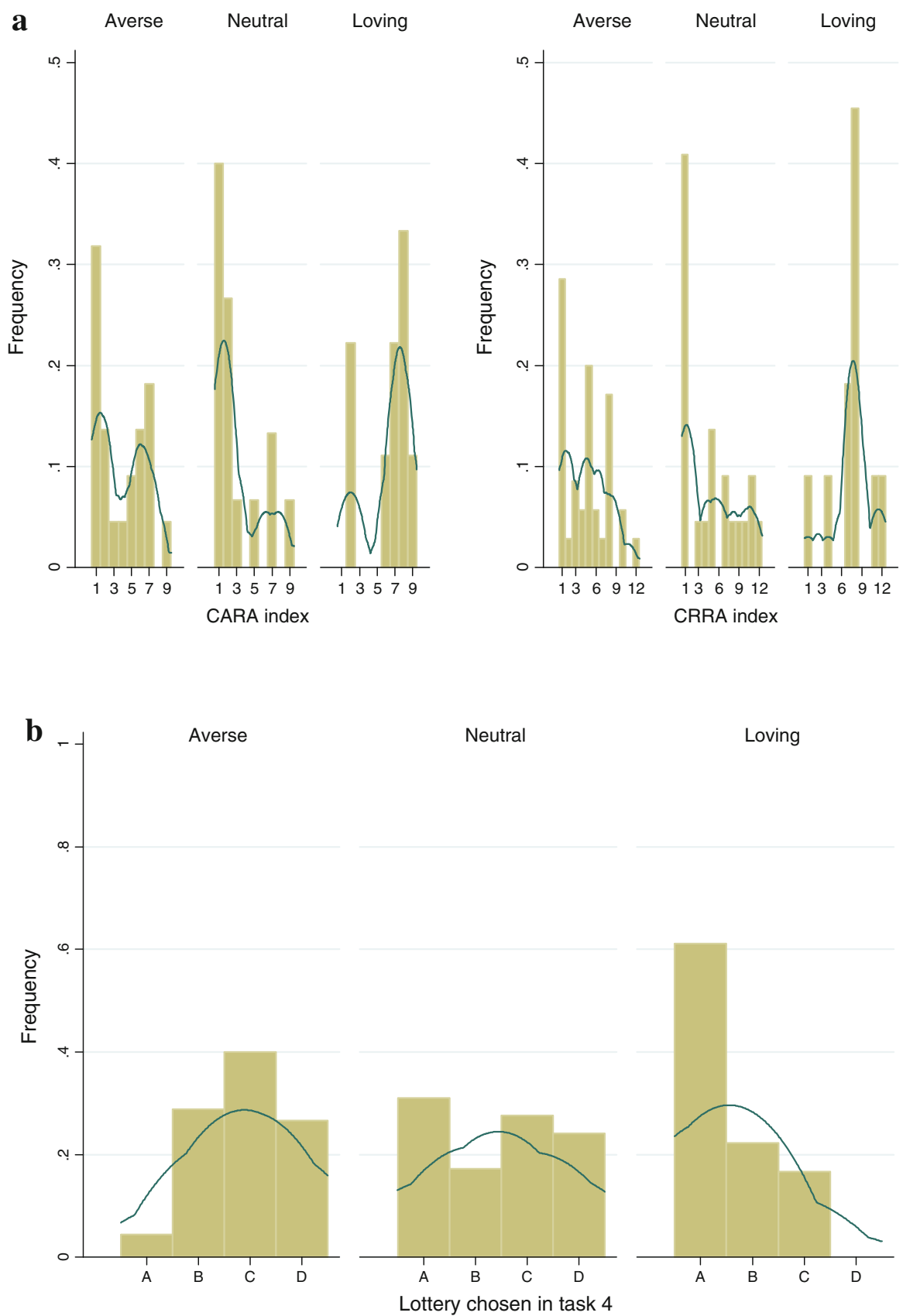

Fig. 2 Risk-attitude orderings in tasks 1-4 and lottery chosen in task 4 by ambiguity attitude

This section will be concluded by analyzing the relation between the sign of the ambiguity attitude and the degree of risk aversion. Figure 2 a shows - through histograms and kernel density plots - that both the distribution of CARA indexes and the 
distribution of CRRA indexes differ according to the sign of the ambiguity attitude. Indeed, under both specifications, the modal risk-aversion index for ambiguity-averse and for ambiguity-neutral is 1 (non-risk-averse subjects), while the modal index for ambiguity-loving subjects is 8 (risk-averse subjects). Differences in the distributions of our risk-aversion ordering among different signs of the ambiguity attitude are indeed found to be significant. ${ }^{24}$ This supposed negative correlation between the sign of the ambiguity attitude and the index of risk aversion is confirmed by rank correlation tests for ambiguity-neutral subjects under $C A R A$ (coeff. $=-0.28, P$ value $=0.063$ ) and for ambiguity-loving subjects under both specifications (under $C A R A$ : coeff. $=-0.39$, $P$ value $=0.007$; under $C R R A$, coeff. $=-0.30, P$ value $=0.013)$. Such negative correlation is not found to be significant for ambiguity-averse subjects. However, if ambiguity-averse subjects are disentangled according to Definition 1 and Definition 2, the negative correlation between the fact of being choice-ambiguity-averse and the index of risk aversion is found to be significant (under $C A R A$ : coeff. $=-0.30, P$ value $=0.029$; under $C R R A$ : coeff. $=-0.31, P$ value $=0.007)$. This last result is even more relevant if one recalls that choice-ambiguity aversion was elicited (tasks 4 and 10) by relying on the same method used to elicit risk aversion (tasks 1-4). Figure $2 \mathrm{~b}$ seems to confirm our intuition: very few ambiguity-averse subjects (less than $5 \%$ ) choose the least risky lottery in task 4 , while the vast majority of ambiguity-loving ones $(61 \%)$ choose this lottery in task 4; moreover, none of the latter chooses the riskiest lottery in task 4. Differences in the distribution of $l_{4}^{j_{4}}$ among the three groups of subjects averse, neutral, and prone to ambiguity are found to be significant. ${ }^{25}$

This point can be further clarified by bringing into the analysis the subject's guess on the number of balls linked to the highest of the two lottery outcomes (henceforth, "winning balls") in task 6 , that could be interpreted as the subject's modal belief on the composition of the small unknown urn. This guess may have influenced the certainty equivalent in task 6 and so the sign of value-ambiguity aversion. ${ }^{26}$ Figure 9 in the Appendix reports the distribution of subjects' guess on the number of winning balls in tasks 6-9 disentangled by the sign of their ambiguity attitude (averse, neutral, and loving). ${ }^{27}$ By looking at the upper-left part of Fig. 9, one can notice that also the

\footnotetext{
24 In order to verify the equality in the distribution of each risk-aversion index among different signs of the ambiguity attitude, two different tests have been performed: Kruskal-Wallis equality-of-populations rank test and Kolmogorov-Smirnov equality-of-distributions test. Both under CARA and under CRRA, the null hypothesis of equality in distributions can be rejected according to the Kruskal-Wallis equality-ofpopulations rank test (respectively for $C A R A$ and $C R R A: P$ value $=0.010, P$ value $=0.010$ ). By performing the Kolmogorov-Smirnov equality-of-distributions test with a pairwise comparison between different signs of the ambiguity attitude, the results are found to be consistent with the Kruskal-Wallis test.

25 Again, a Kruskal-Wallis test has been performed to check whether the distribution of $l_{4}^{j_{4}}$ is different by treatment. According to this test, the null hypothesis of equality in distribution $(P$ value $=0.000)$ can be rejected. A Kolmogorov-Smirnov test has also been performed with a pairwise comparison between different signs of the ambiguity attitude, and the results are consistent with the Kruskal-Wallis test.

26 The guess in task 6 is positively correlated (coeff. $=0.20, P$ value $=0.045$ ) with the "normalized" $C E\left(L_{6}\right)$. In Sect. 4.4 , the specific meaning of "normalized" certainty equivalent is explained.

27 Recall that each unknown small urn in tasks 6-9 has 10 balls inside. Hence, the set of possible guesses in each of these tasks is $\{0,1, \ldots, 10\}$.
} 
distribution of subjects' guess in task 6 is significantly different among different signs of the ambiguity attitude. ${ }^{28}$

Indeed, with a multivariate regression analysis it is found that - controlling or not for treatment effect-both $l_{4}^{j_{4}}$ and the guess in task 6 are highly significant (respectively, $P$ value $=0.000$ and $P$ value $=0.001$ ): the former has a positive effect on being ambiguity-averse, while the latter has a negative one.

About the positive effect of $l_{4}^{j_{4}}$, it may be thought that subjects have made some kind of hedging between the higher risk they accepted through the choice of a risky lottery in task 4 and the ambiguous second-order probabilities over this (second-stage) lottery in task 10. Indeed, if value-ambiguity aversion and choice-ambiguity aversion are disentangled, a positive and highly significant correlation is found between $l_{4}^{j_{4}}$ and each measure of ambiguity attitude: respectively, coeff. $=0.24(P$ value $=0.023)$ for value-ambiguity aversion and coeff. $=0.42(P$ value $=0.000)$ for choice-ambiguity aversion.

About the negative effect of the guess of the number of winning balls in task 6 , this plays a role through a decrease of $C E\left(L_{6}\right)$ and $j_{10}$. Section 4.4 will show that the distribution of subjects' guess in task 6 does not depend on the treatment where the subjects have participated, i.e., on the different distributions of secondorder probabilities that have been generated through our experimental design.

\subsection{Test of the main theoretical predictions}

This section reports and comments on the percentage of classified subjects who satisfy the theoretical predictions stated in Sect. 3. Classified subjects are disentangled according to the sign of their ambiguity attitude (averse, neutral, and loving) and according to the treatment where they participated. All the theoretical predictions tested in this section should hold independent of the treatment. A first set of theoretical predictions (Proposition 5) state different conditions for ambiguity-averse, ambiguity-neutral, and ambiguity-loving subjects. A second set of theoretical predictions (Proposition 6) should hold whatever the sign of the ambiguity attitude.

Figure 3 summarizes the percentage of subjects classified according to Definition 3 whose behavior in tasks 5-7 complies with Proposition 5. In particular, for $78 \%$ (35/45) of ambiguity-averse subjects $C E\left(L_{5}\right) \geq C E\left(L_{7}\right) \geq C E\left(L_{6}\right)$; for $86 \%(25 / 29)$ of ambiguity-neutral subjects $C E\left(L_{5}\right)=C E\left(L_{7}\right)=C E\left(L_{6}\right)$; for $78 \%$ (14/18) of ambiguity-loving subjects $C E\left(L_{5}\right) \leq C E\left(L_{7}\right) \leq C E\left(L_{6}\right)$. Although the percentage of subjects fulfilling predictions of Proposition 5 is higher among the ambiguity-neutral ones, the validity of said predictions does not depend on the sign of the ambiguity attitude. On the other hand, there is a weak dependence on the treatment. Through a $t$ test, it is found that the percentage of subjects complying with Proposition 5 is almost significantly higher in the Binomial $(P$ value $=0.124)$ and in the Unknown

\footnotetext{
28 Through the Kruskal-Wallis test, we can reject the null hypothesis of equality in distribution $(P$ value $=0.000)$. The Kolmogorov-Smirnov test confirms this result.
} 


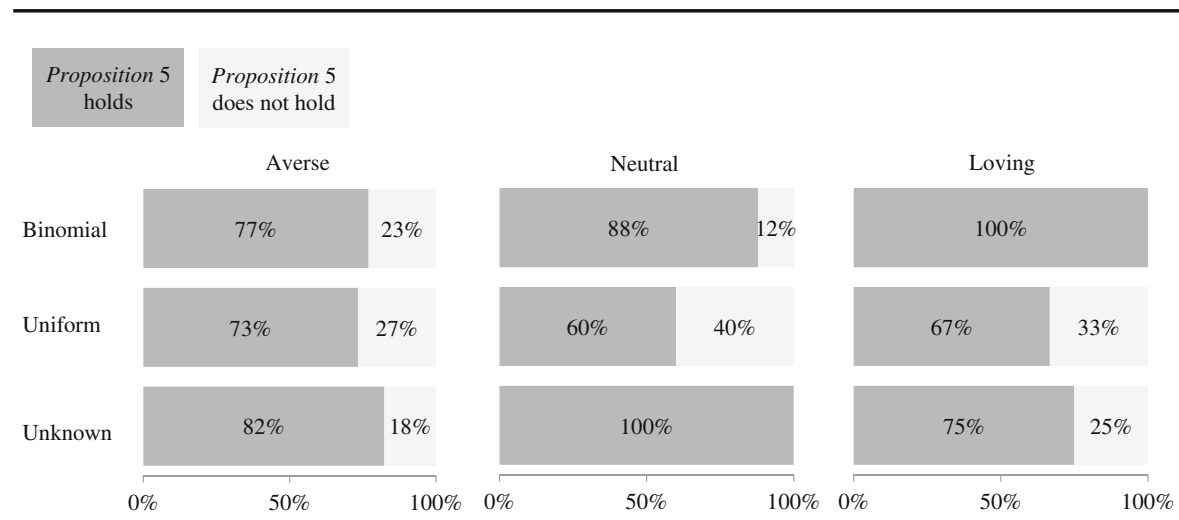

Fig. 3 Percentage of subjects satisfying Proposition 5, by treatment and ambiguity attitude

$(P$ value $=0.120)$ than in the Uniform treatment. Finally, this compliance is uncorrelated with the subject's CARA or CRRA ordering, as predicted by Proposition $5 .^{29}$

Figure 4 reports the percentage of subjects whose behavior in tasks 6-9 complies with Proposition 6 with regard to $C E\left(L_{6}\right)$ (Fig. 4a) and with regard to $C E\left(L_{7}\right)$ (Fig. 4b), respectively. Recall that Proposition 6 provides the same prediction for all subjects being classified according to Definition 3, whatever the sign of their ambiguity attitude and their degree of ambiguity aversion. It is easy to notice that the percentage of subjects fulfilling Proposition 6 is even higher than for Proposition 5 and that it does not depend on which of the two reference certainty equivalents have been applied, $C E\left(L_{6}\right)$ or $C E\left(L_{7}\right)$. In both cases, more than $90 \%$ of ambiguity-averse subjects $(42 / 45$ for $C E\left(L_{6}\right), 41 / 45$ for $\left.C E\left(L_{7}\right)\right)$, all ambiguity-neutral subjects (29/29), and more than $70 \%$ of ambiguity-loving subjects (13/18) comply with Proposition 6. As further proof of "rational" behavior of this huge percentage of subjects, it should be noted that there are only 4/84 subjects satisfying Proposition 6 with regard to $C E\left(L_{6}\right)$ and not satisfying it with regard to $C E\left(L_{7}\right)$; there are only $3 / 83$ subjects who fulfill the predictions with regard to $C E\left(L_{7}\right)$ and not with regard to $C E\left(L_{6}\right)$.

There are no significant differences by treatment if $C E\left(L_{6}\right)$ is taken as reference. If instead $C E\left(L_{7}\right)$ is taken as reference, the percentage of subjects being consistent with Proposition 6 is slightly higher in the Binomial than in the Uniform treatment, with such difference being significant $(P$ value $=0.055)$. On the other hand, whatever the reference certainty equivalent, $C E\left(L_{6}\right)$ or $C E\left(L_{7}\right)$, a significant difference is found by the sign of the ambiguity attitude. Indeed, the percentage of ambiguity-loving subjects complying with Proposition 6 is significantly lower than the percentage of ambiguityaverse ones ${ }^{30}$ and the percentage of ambiguity-neutral ones ${ }^{31}$. Again, compliance with the prediction of Proposition 6 is uncorrelated with the subject's CARA or CRRA ordering, in line with the theory. ${ }^{32}$

\footnotetext{
$29 P$ value $=0.546$ for $C A R A, P$ value $=0.841$ for $C R R A$.

$30 P$ value $=0.023$ for $C E\left(L_{6}\right), P$ value $=0.054$ for $C E\left(L_{7}\right)$.

$31 P$ value $=0.002$ for both $C E\left(L_{6}\right)$ and $C E\left(L_{7}\right)$.

32 If $C E\left(L_{6}\right)$ is taken as reference, $P$ value $=0.202$ for $C A R A, P$ value $=0.278$ for $C R R A$. If $C E\left(L_{7}\right)$ is taken as reference, $P$ value $=0.216$ for $C A R A, P$ value $=0.333$ for $C R R A$.
} 

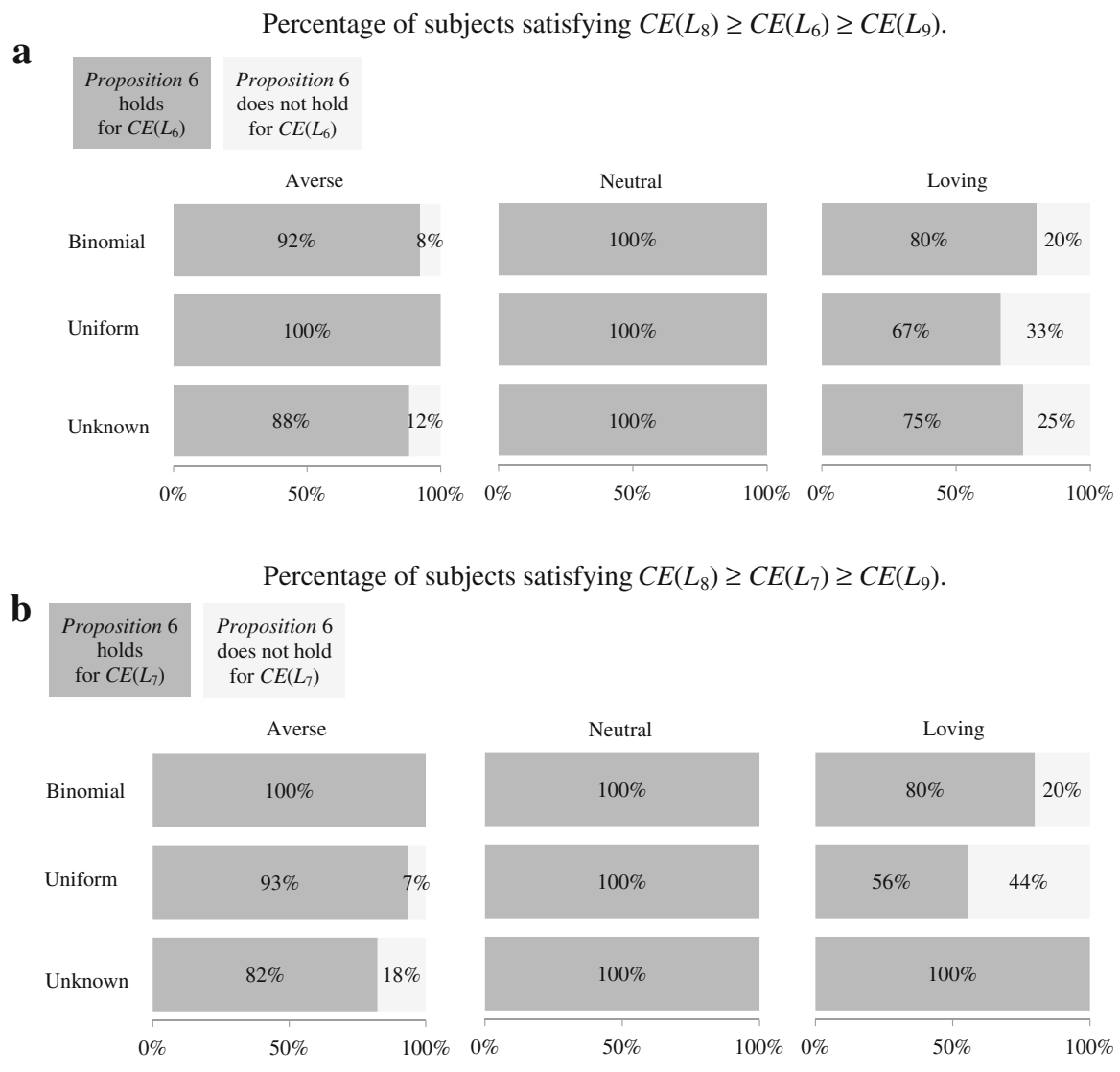

Fig. 4 Percentage of subjects satisfying Proposition 6, by treatment and ambiguity attitude

Figure 5 shows the percentage of classified subjects whose behavior in tasks 5-9 satisfies both Proposition 5 and Proposition 6 at the same time. As seen in Sect. 3, the two propositions taken together lead to relation (5) for ambiguity-averse, relation (6) for ambiguity-neutral, and relation (7) for ambiguity-loving subjects. Indeed, more than $75 \%$ of classified subjects (70/92) state their certainty equivalents in tasks 5-9 in a way that all the "rationality" constraints imposed by the $K M M$ model are satisfied. It is interesting that between Proposition 5 and Proposition 6 the former cuts much more observations, given that percentages of verification in Fig. 5 are only slightly lower than those in Fig. 3. In addition, ambiguity-loving subjects have a lower ratio of fulfillment of Propositions 5 and 6 taken together $(61 \%, 11 / 18)$ than ambiguity-averse subjects $(76 \%, 34 / 45)$ and ambiguity-neutral ones $(86 \%, 25 / 29)$, although the former difference is not significant, possibly due to the low number of ambiguity-loving subjects in our sample. It should also be noted that all ambiguity-neutral subjects fulfilling Proposition 5 also fulfill Proposition 6.

Furthermore, significant differences both by the sign of the ambiguity attitude and by treatment are encountered. About the former, we have anticipated above that the percentage of ambiguity-loving subjects complying with both propositions is found 


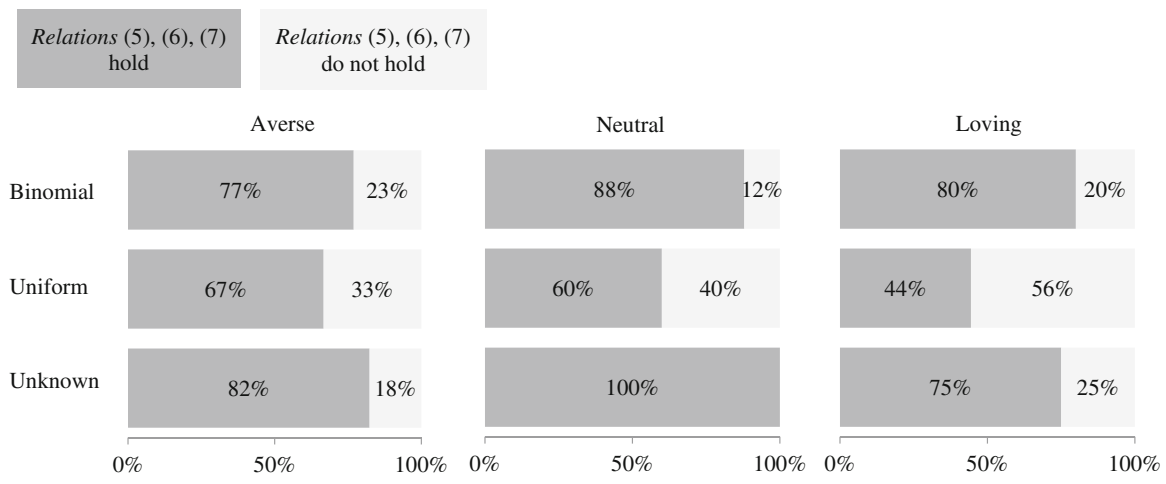

Fig. 5 Percentage of subjects satisfying (5), (6), and (7), by treatment and ambiguity attitude. [Note Relations (5), (6) and (7) refer respectively to ambiguity-averse, ambiguity-neutral and ambiguityloving subjects]

Fig. 6 Disentanglement of low, medium, and high ambiguity aversion w.r.t. $C E\left(L_{8}\right) \gtreqless C E\left(L_{5}\right)$ and proneness w.r.t. $C E\left(L_{5}\right) \gtreqless C E\left(L_{9}\right)$

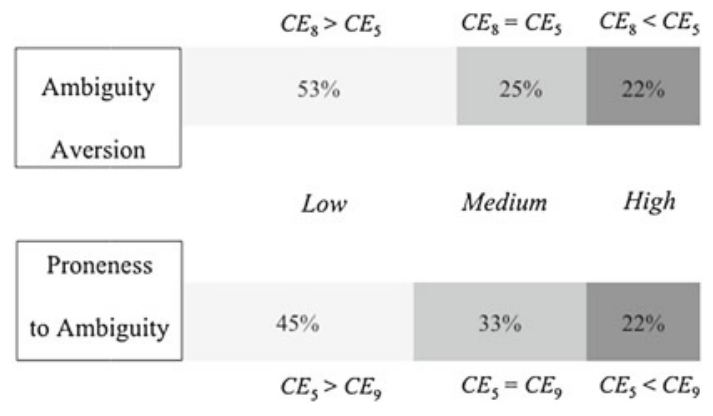

to be significantly lower than the percentage of ambiguity-neutral ones ( $P$ value $=$ 0.038). About the latter, the percentage of subjects complying with both propositions is significantly higher in the Binomial $(P$ value $=0.038)$ and in the Unknown $(P$ value $=0.018$ ) than in the Uniform treatment. It is particularly striking that in the Unknown treatment, all subjects satisfying Proposition 5 satisfy also Proposition 6, both with regard to $C E\left(L_{6}\right)$ and with regard to $C E\left(L_{7}\right)$. In fact, percentages of compliance with the theoretical predictions are the same in Figs. 3 and in 5, whatever the sign of the ambiguity attitude. Once more, compliance with the entire set of our theoretical predictions is uncorrelated with the subject's CARA or CRRA index. ${ }^{33}$

Finally, the subject's behavior will be analyzed in the only two tasks for which no sharp theoretical prediction is available. Reference here is made to tasks 5 and 8 for ambiguity-averse subjects and to tasks 5 and 9 for ambiguity-loving ones. From relation (6), in the limit case of smooth ambiguity-neutrality, $C E\left(L_{8}\right)>C E\left(L_{5}\right)$ and $C E\left(L_{5}\right)>C E\left(L_{9}\right)$ must hold. Therefore, in Fig. 6, ambiguity attitude is classified as "Low" for those ambiguity-averse subjects and for those ambiguity-loving ones who behave like the ambiguity-neutral ones, respectively, in tasks 5 and 8 and in tasks 5 and 9. "Medium" and "High" ambiguity attitudes are classified accordingly, that is a

$33 P$ value $=0.838$ for $C A R A, P$ value $=0.765$ for $C R R A$. 
subject is highly-ambiguity-averse if $C E\left(L_{8}\right)<C E\left(L_{5}\right)$ and highly-ambiguity-loving if $C E\left(L_{5}\right)<C E\left(L_{9}\right)$.

Surprisingly enough, the same percentage of highly-ambiguity-averse (10/45) and of highly-ambiguity-loving (4/18) subjects is found in our sample. Subjects in the former group prefer to know the composition of the small urn with certainty (task 5) rather than knowing only that the worst scenarios are implausible (task 8). Specularly, subjects in the latter group prefer to know only that the best scenarios are implausible (task 9) rather than knowing the composition of the small urn with certainty (task 5). Notice that neither the sign of $C E\left(L_{8}\right)-C E\left(L_{5}\right)$ for ambiguity-averse subjects nor the sign of $C E\left(L_{5}\right)-C E\left(L_{9}\right)$ for ambiguity-loving ones is correlated with any of the explanatory variables introduced above (treatment, CARA ordering, CRRA ordering, lottery chosen in task 4 , and guess on the winning balls, respectively, in task 8 and in task 9). The effects of all these variables are not significant at all, both in the univariate analysis considering each control singularly and in the multivariate regressions. The only significant result detected is indeed counterintuitive. It is found that $C E\left(L_{8}\right)-$ $C E\left(L_{5}\right)>0$ depends positively on strong choice-ambiguity aversion (i.e., $j_{10} \prec j_{4}$ ). This would lead to conclude that strongly-choice-ambiguity-averse subjects are not so strongly-value-ambiguity-averse $\left(C E\left(L_{6}\right)<C E\left(L_{5}\right)\right)$, thereby explaining why the above found correlation between strong choice-ambiguity-attitude and strong valueambiguity-attitude, although positive and significant, is not so high.

As anticipated at the end of Sect. 3, the combination of Proposition 7 and Corollary 9 suggests a possible treatment effect on the percentage of ambiguity-averse subjects showing $C E\left(L_{5}\right) \geq C E\left(L_{8}\right)$ and on the percentage of ambiguity-loving subjects showing $C E\left(L_{9}\right) \geq C E\left(L_{5}\right)$. Such prediction relies on the assumption of a similar distribution of risk attitude among the three treatments, which is satisfied, as shown in Sect. 4.1. Indeed, the prediction on the treatment effect over the size of the ambiguity attitude is verified. The percentage of medium- and highly-ambiguity-averse subjects is lower in the Binomial $(31 \%, 4 / 13)$ than in the Uniform $(60 \%, 9 / 15)$ and in the Unknown treatment $(47 \%, 8 / 17)$, with the difference being almost significant between the Binomial and the Uniform $(P$ value $=0.131)$. Specularly, the percentage of medium- and highly-ambiguity-loving subjects is lower in the Binomial (40\%, 2/5) than in the Uniform $(67 \%, 6 / 9)$ and in the Unknown treatment $(50 \%, 2 / 4)$.

\subsection{Treatment effects over beliefs and certainty equivalents}

The analysis of the experimental results can be concluded by looking at possible treatment effects over the certainty equivalent and over the guess of winning balls in tasks 6-9.

Corollary 9 states that $C E\left(L_{t}\right)$ for $t=6, \ldots, 9$ should be higher in the Binomial than in the Uniform and in the Unknown treatment. If the subject's guess on winning balls in task $t$ is correlated with $C E\left(L_{t}\right)$ for $t=6, \ldots, 9$, then it should be found that this guess should also be higher in the Binomial treatment. Now, it is true that our experimental design is between-subject; hence, it cannot be directly stated whether and how a subject changes her certainty equivalent (and her guess) according to the way in which the unknown small urn is generated at the beginning of task 6. However, under the assumption that both the distribution of risk attitudes and the distribution 
of ambiguity attitudes are not too different among treatments- that is what has been shown, respectively, in Sect. 4.1 and in Sect. 4.2-possible treatment differences in the distribution of certainty equivalents and of guesses in tasks 6-9 can be analyzed.

Figure 7 reports the distribution of subjects' guess on the number of winning balls in tasks 6-9 disentangled by treatment. The graphs by treatment seem to suggest that the distribution of guesses in the Unknown treatment is close to the one in the Binomial treatment, and both are quite different from the one in the Uniform treatment. This result is in line with the principle of insufficient reason, that should lead a subject in the Binomial and in the Unknown treatment to provide a guess equal to 5 winning balls in tasks 6 and 7, between 6 and 7 winning balls in task 8 , and between 3 and 4 winning balls in task 9. Conversely, in the Uniform treatment, all guesses should be equivalent: any guess over a scenario that is plausible in a specific task is justifiable.

However, it is found that the distribution of a subject's guess in task $t$ is not significantly different among treatments, for $t=6,8,9 .{ }^{34}$ Our intuition is instead correct in task 7. According to the Kruskal-Wallis test on the equality in distribution of guesses by treatment, the null hypothesis can be rejected at a $10 \%$ level $(P$ value $=0.082)$. More precisely, according to the Kolmogorov-Smirnov equality-of-distributions test, there is not equality in the distribution of guesses between the Binomial and the Uniform treatment $(P$ value $=0.003)$ and between the Unknown and the Uniform treatment $(P$ value $=0.016$ ). Also in this specific task, it seems that the subjects in the Unknown treatment state similar guesses to those of the subjects in the Binomial one.

Focusing on the relation between certainty equivalents and subjects' guesses, overall, the former are never found to be correlated with the latter in tasks 6-9 within the same task. ${ }^{35}$

In general, certainty equivalents in task $t\left(C E\left(L_{t}\right)\right)$ are negatively correlated with the $C A R A$ and/or with the $C R R A$ ordering (recall that the ordering index increases with risk aversion). This is reasonable: as shown above, the subject's certainty equivalent elicited in task 5 (and in the following four tasks) relies on the lottery chosen in task 4. This choice also depends on the CARA or the CRRA ordering elicited in tasks 1-4 (as seen in Sect. 4.2, this choice also influences the sign of the ambiguity attitude).

The link between certainty equivalent and risk aversion is further investigated by considering the lottery chosen in task $4\left(l_{4}^{j_{4}}\right)$ and its relation with the "normalized" values of the certainty equivalents, i.e., their index in tasks 6-9, with $C E\left(L_{t}\right)=\underline{x}_{4}^{j_{4}}$ being assigned index 1 and $C E\left(L_{t}\right)=\bar{x}_{4}^{j_{4}}$ being assigned index $11 .{ }^{36}$ Figure 10 in the Appendix shows the "normalized" values of $C E\left(L_{t}\right)$ for $t=6, \ldots, 9$. Once $C E\left(L_{t}\right)$ are normalized, they are still found to be correlated with the risk-attitude index.

\footnotetext{
34 According to the Kruskal-Wallis test on the equality in distribution of guesses by treatment, the null hypothesis cannot be rejected (respectively, for task 6, 8, 9, $P$ value: $0.739,0.375,0.175$ ). The KolmogorovSmirnov equality-of-distributions test with a pairwise comparison between treatments confirms this result. 35 However, $C E\left(L_{6}\right)$ is positively correlated (coeff. $=0.35$ ) with the guess in task 6 only in the Uniform treatment $(P$ value $=0.037)$. Also, $C E\left(L_{9}\right)$ is positively correlated (coeff. $\left.=0.36\right)$ with the guess in task 9 only in the Binomial treatment $(P$ value $=0.035)$.

36 In tasks 5-9, each subject always has the possibility to choose among eleven possible selling prices. Therefore, for every $t=5,6, \ldots, 9$, index 1 can always be assigned to $C E\left(L_{t}\right)=\underline{x}_{4}^{j_{4}}$, index 11 to $C E\left(L_{t}\right)=\bar{x}_{4}^{j_{4}}$ and internal $C E\left(L_{t}\right)$ can be indexed accordingly. See footnote 5 .
} 

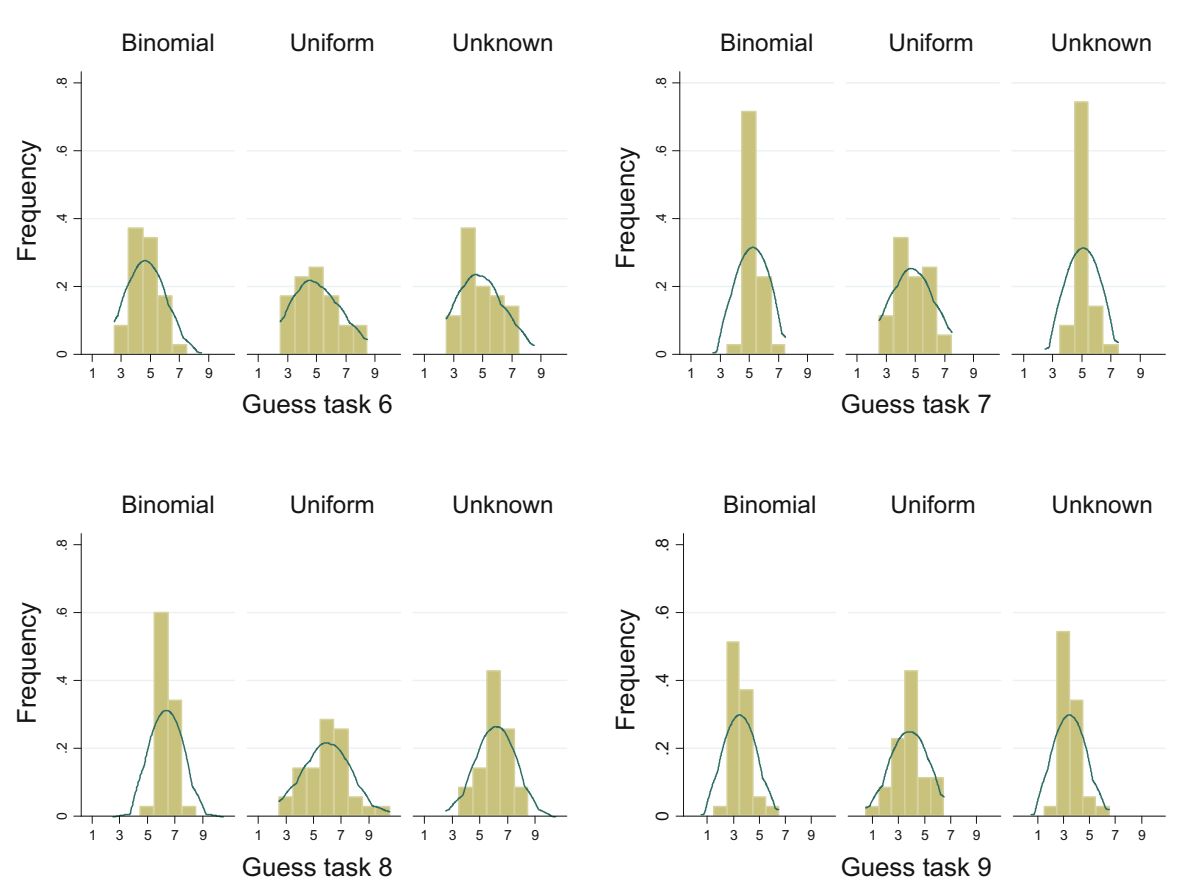

Fig. 7 Distribution of subjects' guess on the number of "winning balls" in tasks 6-9 by treatment

However, while the relation between risk aversion and $C E\left(L_{6}\right), C E\left(L_{7}\right)$ and $C E\left(L_{8}\right)$ is negative, $C E\left(L_{9}\right)$ instead shows a positive relation with risk aversion. The last result can be referred to the positive correlation between risk aversion and ambiguity proneness highlighted in Sect. 4.2. Further, as long as the distribution of the secondorder probabilities is symmetric around $\theta=5$ (tasks 6 and 7), the normalized certainty equivalents are correlated with the guesses in the same task. Such correlation is not found in tasks 8 and 9.

A strong regularity is that, by regressing either $C E\left(L_{t}\right)$ or its normalized version over the guess in the same task, the index of risk attitude and the treatment, no significant treatment effects are found for any $t=6,7,8,9 .{ }^{37}$ As one can notice from Fig. 10, the differences among treatments in the distribution of normalized $C E\left(L_{t}\right)$ are not statistically significant for every $t=6, \ldots, 9$. In addition, the sign of all differences $C E\left(L_{t+1}\right)-C E\left(L_{t}\right)$ for $t=5,6,7$ and $C E\left(L_{t+2}\right)-C E\left(L_{t}\right)$ for $t=6,7$ do not depend on the treatment. ${ }^{38}$

\footnotetext{
37 A relevant exception is again represented by the (normalized) certainty equivalent in task 7 . Controlling for CARA and treatment and taking the Binomial as reference treatment, the Unknown treatment has a positive and significant effect $(P$ value $=0.059)$ over $C E\left(L_{7}\right)$. Controlling for $C R R A$ and treatment, the Uniform treatment also has a positive and significant effect $(P$ value $=0.041)$ over $C E\left(L_{7}\right)$.

38 To be more precise, only in the regressions for $C E\left(L_{7}\right)-C E\left(L_{6}\right)>0$ (CE normalized), the Uniform treatment significantly increases the probability that $C E\left(L_{7}\right)-C E\left(L_{6}\right)>0$ with respect to the Binomial treatment $(P$ value $=0.040)$. This result also holds when controlling for the difference in the guesses about the number of winning balls in task 7 and task 6 .
} 
All these findings lead to the following conclusion. Despite some differences in the distribution of guesses among treatments, no treatment effect is found on the distribution of certainty equivalents in the ambiguous tasks. Therefore, the only significant difference among treatments is the one shown at the beginning of Sect. 4.2 about the distribution of $\left(C E\left(L_{6}\right)-C E\left(L_{5}\right)\right)$ if combined with the distribution of $\left(j_{10}-j_{4}\right)$, i.e., the two conditions leading to state a subject's coherent-ambiguity attitude.

\section{A critical discussion vis-a-vis the experimental literature on ambiguity aversion}

This section will attempt to frame this contribution into the experimental literature on ambiguity aversion. An overview of previous contributions in the literature that are relevant to this paper is presented in order to support our experimental design and to compare experimental results. To afford maximum clarity, this section has been divided into four sub-sections, each treating different but complementary issues. The first subsection will focus on the difference among risk, uncertainty, and ambiguity so as to clarify the definition given to each of these terms. Second, the issue of designing experiments to discriminate between theories of ambiguity as well as to operationalize one theory will be discussed. This study belongs to the latter category and evaluates the structure of (risk and) ambiguity attitudes within the $K M M$ model. Third, the experimental methods used to elicit risk and ambiguity will be looked at critically, taking into account previous experimental tests of these instruments. Finally, the fourth sub-section will compare our results with previous findings in the literature.

\subsection{Risk, uncertainty, and ambiguity: distinguishing close concepts}

The term "uncertainty" is often used as synonymous of both risk and ambiguity even though these two terms identify different situations. The fundamental difference between risk and ambiguity concerns the distinction between whether probability is known or unknown. This distinction can take different names: "measurable" uncertainty or risk, with known probabilities, and "unmeasurable" uncertainty or ambiguity, with unknown probabilities (Knight 1921); "precise" versus "vague" probability (Savage 1954); "unambiguous" versus "ambiguous" probability (Ellsberg 1961); and so forth. Espousing the general definition of risk, Harrison (2011) also provides a distinction between uncertainty and ambiguity based on the availability of relevant information to form subjective belief distribution: "When we have to worry about the underlying nondegenerate distribution, when reduction of compound lotteries is not assumed, then we have moved from the realm of (subjective) risk to uncertainty. And, when the individual does not even have enough information to form any subjective belief distribution, degenerate or non-degenerate, we are in the realm of ambiguity" (p. 352). However, in the literature, the term "ambiguity" is also often introduced when the individual forms a certain subjective belief distribution, but she faces "situations in which some events do not have an obvious, unanimously agreeable, probability assignment" (Ghirardato 2004, p. 36). ${ }^{39}$

39 Ghirardato (2004) also discusses the issue of the formal definition of ambiguity and ambiguity attitude. 
Therefore, according to Harrison (2011), the KMM model pertains to the "measurable" uncertainty or risk realm. Consequently, all decision tasks of treatments 1-2 and decision tasks $1-5$ of treatment 3 deal with risk and uncertainty. Only decision tasks 6-10 of treatment 3 refer to ambiguity. However, if the term "ambiguity" is also associated to the case where the reduction of compound lottery axiom does not apply, then also decision tasks $6-10$ of treatments $1-2$ will also refer to ambiguity. This is the meaning given to the term "ambiguity" within $K M M$ and in this paper too.

\subsection{Discriminating between theories versus operationalizing one theory}

This sub-section reviews two broad classes of empirical contributions in decision making under ambiguity. The first class includes previous empirical works aimed at designing experiments to discriminate between ambiguity models. These can be referred to as "horse race" tests. The second class includes empirical contributions aimed at operationalizing one model of ambiguity aversion. Within this latter class, only studies implementing a $K M M$-type experimental environment are reviewed here.

Within the first class, several papers have empirically investigated the descriptive and predictive power of theories of decision making under ambiguity. ${ }^{40}$ Some of them have studied ambiguity attitude by purposefully excluding two-stage probability models. ${ }^{41}$ Complementary to these, some other papers (e.g., Conte and Hey 2013) investigated ambiguity attitude considering only two-stage probability models. Some others have produced experimental designs aimed at comparing the performance of $K M M$ with that of non-expected utility models (Halevy 2007; Ahn et al. 2011). Halevy (2007) suggests that there is no unique theoretical model that universally captures ambiguity preferences. $^{42}$ In the same line, Conte and Hey (2013) compare the performance of different theoretical decision models-expected utility, $K M M$, rank-dependent expected utility, and Alpha model of Ghirardato et al. (2004) — through an experimental design slightly different from Halevy's (2007). In fact, even though these two studies are similar for what concerns the implementation of ambiguity in the laboratory, they present some differences in the methodology. ${ }^{43}$ Conte and Hey's (2013) results suggest that KMM performs best among the four tested models. ${ }^{44}$ However, not all exper-

\footnotetext{
40 Early literature is surveyed in Camerer and Weber (1992) and Camerer (1995).

41 See for example Hey et al. (2010) and Hey and Pace (2014): both experimental designs are aimed at testing only non-two stage probability models.

42 In particular, Halevy (2007) found that $15-20 \%$ of his subjects are ambiguity-neutral and able to reduce compound lotteries. Another $35 \%$ of subjects exhibit ambiguity aversion (proneness) together with aversion (proneness) to mean-preserving spreads in the second-order distribution. Both these categories of subjects are consistent with $K M M$.

43 Indeed, both papers use Ellsberg-type urns. In particular, Conte and Hey (2013) adopted the same urns 2 and 3 as Halevy (2007) that reproduce two-stage lotteries. However, Conte and Hey (2013) use exclusively pairwise questions to reduce the number of parameters to be estimated, no meaning to estimate a utility function. Differently, Halevy (2007) asks subjects to state certainty equivalents through the $B D M$ mechanism so as to infer a utility function from the subject's answers.

44 The authors found results in favor of $K M M$ both through individual estimates (56\% of subjects show behavior consistent with $K M M$ ) and by classifying subjects according to posterior probabilities of each of them being coherent with one out of four types of preferences (50\% for $K M M)$.
} 
imental studies offer support for KMM. Ahn et al. (2011) found a result opposite to Conte and Hey's (2013) by performing a portfolio choice experiment ${ }^{45}$ aimed at investigating rank-dependent theories versus smooth ambiguity. Their tests of significance suggest that the majority of subjects are well described by the subjective expected utility model. Moreover, among the remaining subjects, $K M M$ fails to explain the behavior of those showing ambiguity aversion.

Thus far, not many experiments have been designed with the explicit purpose of testing behavioral predictions within the KMM model only. Chakravarty and Roy (2009) is one of them. They have tried to separate attitude toward risk from that toward ambiguity using the multiple price list method as the only experimental instrument. Their main objective consisted of investigating potential differences in subjects' behavior under uncertainty over gains versus uncertainty over losses. They found a positive correlation between risk attitude and ambiguity attitude in the domain of gains (although, in the aggregate, subjects are risk-averse and ambiguity-neutral). Their result is not sole. Indeed, many empirical studies have found such positive correlation; ${ }^{46}$ only few have no correlation whatsoever. ${ }^{47}$ Within this class of studies, Andersen et al. (2009) is the only experimental work that clearly states that the correlation between risk attitude and ambiguity attitude can be negative. They estimated attitudes toward ambiguity, attitudes toward risk, and subjective probabilities in a rigorous manner within a $K M M$ type model, by making some parametric assumptions about the form of the distribution of the priors and the uncertain process. They found subjects who are risk-averse, and at the same time ambiguity-loving.

A new approach, which is becoming popular in experimental economics, is based on adopting mixture models. These models have an important advantage with respect to subject-by-subject estimation through competing models. Heterogeneity across individuals - a common finding of experimental studies on decision making under risk and ambiguity - is captured within a unique model, which merges different theories explaining the behavior of different subjects in the same sample. Several papers have adopted this approach in decision making under risk. Cohen et al. (2011) consider $E U$ and cumulative prospect theory (Tversky and Kahneman 1992); they also include a discussion of the previous use of mixture models in economics. Conte et al. (2011) consider $E U$ and rank-dependent expected utility. More recently, this approach has also been adopted in decision making under ambiguity. Conte and Hey (2013), along with a subject-by-subject estimation of individuals' preference functionals, estimated a mixture of $E U, K M M$, rank-dependent expected utility and Alpha model (Ghirardato et al. 2004).

From a methodological point of view, these mixture models can be interpreted as a hybrid of the two classes of empirical contributions presented above, i.e., those discriminating between theories and those operationalizing one theory.

\footnotetext{
45 Ahn et al. (2011) implemented an experimental design where subjects are asked to choose between different lotteries that duplicate the return of a portfolio containing a safe asset and an ambiguous asset.

46 Among several studies finding a positive correlation, see Lauriola and Levin (2001), Di Mauro and Maffioletti (2004), Bossaerts et al. (2010), and Ahn et al. (2011).

47 Among the few detecting no correlation, see Cohen et al. (1987) and Cohen et al. (2011).
} 
Among those studies fitting none of the three categories above, a relevant one for comparisons with this paper is Abdellaoui et al. (2011), which investigates the relationship between attitude toward risk, compound lotteries and ambiguity in a model-free setting.

\subsection{Strengths and weaknesses of our instruments}

This subsection will critically evaluate the risk and ambiguity elicitation methods used in this study. In particular, discussion will revolve around the flexibility of such instruments in coping with other questions not covered in this study.

It should be recalled that risk attitude was elicited through both a portfolio choice method (tasks 1-4) and a BDM mechanism (task 5).

It has been highlighted above that risk-attitude elicitation through portfolio choices (tasks 1-4) here carried out gives results similar to the more standard Holt and Laury (2002) method. If on the one hand this corroborates our first risk-elicitation instrument, on the other hand it raises doubt as to whether such instrument may suffer from the same "order effects" problem of Holt and Laury (2002). In fact, moving from task 1 to task 4, the subject's "initial wealth" is non-decreasing (see Table 3). Further, in tasks 3-4, the lotteries proposed always have an expected value higher than those in tasks 1-2 (almost twice on average, as shown in Table 7 in the Appendix). Harrison et al. (2005) demonstrated and Holt and Laury (2005) confirmed the possibility of order effects by scaling up real payments by 10 or 20 times. In our design, this might not represent a problem, given that the scale from tasks $1-2$ to tasks $3-4$ is very low (1.8 times on average). However, our design is easily adaptable to check for order effects. The order of presentation of tasks 1-4 may be inverted in some of the experimental sessions, and possible differences in the distribution of the risk-aversion parameter between tasks 1-4 sessions and tasks 4-1 sessions may be investigated.

Our experimental design may be further refined to disentangle risk-neutral subjects and risk-loving ones, which in Tables 4 and 5 are all assigned (respectively, CARA and $C R R A$ ) index 1 . This will only require in tasks $1-4$ some slight alterations in the set of possible lotteries in Table $2 .^{48}$

The risk-attitude elicitation in task 5 also involves some reliability problems, pointed out in the literature by both experimental economists and theorists.

From an experimental point of view, Plott and Zeiler (2005) have expressed many concerns with the way that the $B D M$ mechanism is popularly implemented. Our instructions and implementation of the $B D M$ minimize these potential problems by taking into account almost all of the features of Plott and Zeiler's (2005) suggested

\footnotetext{
48 For example, the set of possible lotteries $l_{t}^{j}$ (with $j=A, B, C, D$, and $t=1,2,3,4$ ) in Table 2 may be enriched with just one lottery in task 4 , namely $l_{4}^{E}=(40,0.5 ; 0,0.5)$. Then, under the $C R R A$ specification, subjects with $R R A \in(-0.146,0.123)$ would pick $\left(l_{1}^{j_{1}}, l_{2}^{j_{2}}, l_{3}^{j_{3}}, l_{4}^{j_{4}}\right)=(D, D, D, D)$ and subjects with $R R A \in(-\infty,-0.146)$ would pick $\left(l_{1}^{j_{1}}, l_{2}^{j_{2}}, l_{3}^{j_{3}}, l_{4}^{j_{4}}\right)=(D, D, D, E)$. Notice that this would lead to disentangle risk-neutral and risk-loving subjects as in Table 3 (p. 1649) of Holt and Laury (2002).
} 
procedure. ${ }^{49}$ In this respect, to minimize the confusion effect, ${ }^{50}$ before task 5 of our experiment, subjects have received a thorough explanation of our BDM mechanism together with some numerical examples. ${ }^{51}$ Furthermore, as extensively underlined in Sect. 2, a physical randomizing device is used to encourage each subject to see the random buying price for her lottery (i.e., the one randomly selected by the "drawer") as independent of her stated selling price. This also held for tasks 6-9, where the $B D M$ was used for ambiguity-attitude elicitation. Finally, anonymity in decisions and in earnings was ensured. The similarities between our implementation of the $B D M$ and the one in Plott and Zeiler (2005) may have prevented the endowment effect. In fact, Plott and Zeiler (2005) have found it to be insignificant, when sufficient controls have been introduced in the $B D M$ implementation. ${ }^{52}$

From a theoretical point of view, several researchers (e.g., Holt 1986) have pointed out that, when preferences do not satisfy the axioms underlying $E U$ theory, the $B D M$ mechanism may not elicit valuations accurately. In this regard, Karni and Safra (1987) have shown that the certainty equivalent of a lottery elicited using the $B D M$ mechanism respects the preference ordering if and only if preferences satisfy the independence axiom. This is assumed in our theoretical analysis, as we rely on $K M M$ in choice under ambiguity and as a consequence on $E U$ in choice under risk.

About our ambiguity-elicitation instruments, an important issue raised in the literature is subjects' thinking about strategic behavior and/or manipulations by the experimenter in Ellsberg-type tasks (Schneeweiss 1973; Kadane 1992). At the end of Sect. 2, we have presented all the features of the experimental design introduced to avoid such problems. First, all our instruments are physical instruments, and we never rely on computer-generated realizations of random processes. Second, all subjects have the chances to check any randomizing instrument before its implementation. Further, the randomization was handled in a manner so as to ensure that subjects did not assume that the experimenter was picking numbers and/or rearranging urns composition after "knowing" what the subjects choices were. For example, lotteries' random buying prices in the $B D M$ in tasks 5-9 were drawn once for all these tasks before task 5 began. In treatment 1 and treatment 3, the composition of the unknown small urn used for tasks 6-10 was randomly determined before the beginning of task 6 (see Fig. 1). Both the unknown small urns (treatment 1 and treatment 3 ) and the transparent construction urn (treatment 2) were left on a table in front of the experimental

\footnotetext{
49 See Harrison and Rutström (2008) for a deeper analysis of these and additional issues, as well as alternative elicitation methods. They also review useful techniques to estimate behavioral errors that could arise in the calculation of reservation prices through the $B D M$ mechanism.

50 An important practical problem of each $B D M$ mechanism is that it is complicated, and if subjects fail to understand it, the elicited values might reflect their confusion and not their valuation.

51 Differently from what recommended by Plott and Zeiler (2005), we did not introduce any practice round before task 5 . This is because we did not want subjects to have any feedback about random draws of buying prices before going through the BDM mechanism in tasks 5-9. However, before task 5, both the drawer and each subject participating in the experiment were given the chance to check that each of the four envelopes - through which the $B D M$ was physically implemented-contained the eleven numbered tickets here indicated in footnote 5. Further, the fact that instructions of each new task were given prior to that task allowed subjects to focus on a $B D M$ at a time.

52 To test for possible endowment effects, our experimental design should be extended to allow subjects to act as lottery sellers in some tasks (as in our experiment) and as lottery buyers in others.
} 
subjects from task 6 until the end of the experiment, i.e., until they were eventually used. In treatment 3 , an additional random draw was made at the beginning of the experiment to determine the color of the "winning balls" in the unknown small urn. ${ }^{53}$ Finally, all the previous operations were physically carried out by one of the subjects showing up for the experimental session, randomly chosen before its beginning and not participating in the experiment. All these implementation devices were meant to make our subjects aware that no manipulation from the experimenter was possible about the composition of the unknown small urn used for the ambiguous tasks.

Although the way we create "ambiguous" decision settings may seem less natural than alternative physical means in the experimental literature (e.g., the Bingo blower used by Andersen et al. 2009, and by Hey et al. 2010), it is characterized by higher flexibility and lower distortions on the level of ambiguity a subject "objectively" perceives. For example, with a Bingo blower, one cannot physically produce our tasks 6 and 10, characterized by the greatest possible level of ambiguity. In fact, during the rotations of the Bingo blower, subjects cannot count the balls of each color, but they can easily realize that there are balls of each color. Further, looking at its rotations, different subjects in the same session may have a different perception of the level of ambiguity, e.g., depending on the specific attention paid to the rotations and on the physical distance of the subject from the Bingo cage.

One last comment should be made about our payment protocol. In our experiment, only one of the ten decision tasks was randomly selected at the end of the experiment to determine participants' final earnings. Therefore, we use a random-lottery incentive mechanism (henceforth $R L I M$ ) with the twofold goal of obtaining no interaction between different decision tasks and proposing bigger stakes to our experimental subjects. ${ }^{54}$ In recent years, this payment protocol has become the most used incentive system for individual choice experiments, and many studies have used and tested it with success. ${ }^{55}$ However, any payment protocol might in principle create distortions in individual choice experiments, and even RLIM is not exempt from this potential problem, as recent studies have clearly shown. ${ }^{56}$ These recent findings have reported

\footnotetext{
53 See footnote 9 .

54 A possible theoretical criticism of the random-lottery incentive mechanism, put forward by Holt (1986), was demonstrated not to occur empirically by Starmer and Sugden (1991).

55 Among the most recent, see Hey and Lee (2005) and Lee (2008).

56 Cox et al. (2012) have compared the performance of several payment mechanisms in individual choice tasks and found a new mechanism-with the same expected value of payoff incentives as RLIM-that is less biased than RLIM. With this new mechanism, at the end of the experiment, one state of nature is randomly drawn, and then all (comonotonic) lotteries chosen in the experiment are paid out for this state of nature and the payoff divided by the number of tasks. Moreover, Harrison and Swarthout (2012) have shown that preference estimates obtained under RLIM differ from those obtained in a one-task design and, more generally, documented concerns about the use of payment protocols over multiple choices that, as RLIM, assume the validity of the independence axiom. Similar concerns may be ascribed to the fact that the application of RLIM as payment protocol requires no violation of the reduction of compound lotteries axiom (henceforth $R O C L$ ). In this regard, Harrison et al. (2012) have found no violation of ROCL when subjects are presented with only one choice and violation of $R O C L$ when subjects are presented with many choices and RLIM is used as a payment protocol.
} 
distortions linked to the application of RLIM to experiments with several tasks under risk. When unknown lotteries are introduced within one or more tasks (as in this paper), distortions due to the use of RLIM as a payment protocol may be amplified. However, the opposite hypothesis may be equally reasonable in our study: due to the greater "complexity" an ambiguous task involves, the subject may focus more on each single task, as there is a compound lottery even within the task itself. This could lead the subject to give less importance to the way a single task is selected for payment. Further research should explore and clarify this issue.

\subsection{A comparison of our results with similar empirical studies}

Within the two broad classes of empirical contributions in decision making under ambiguity, this paper belongs to the second class: the KMM model is made operational and the structure of risk and ambiguity attitudes is evaluated within this model. However, in this subsection, our study is compared with the closest previous contributions belonging to all groups indicated in Sect. 5.2. In particular, we focus here on the differences in findings.

Within the first group of studies that have produced experimental designs aimed at comparing the performance of $K M M$ to that of non-expected utility models, Halevy (2007) is certainly the closest to our study in terms of experimental design. In fact, we use a similar $B D M$ mechanism to elicit risk attitude and value-ambiguity attitude. However, there are two main differences. On the one hand, compared to Halevy (2007), in our experimental design, the variation of the subject's certainty equivalent is studied for a larger number of ambiguity levels and of distributions of second-order probabilities. Therefore, a richer set of theoretical relations-that a subject's decisions should satisfy to be consistent with $K M M$-is formulated. On the other hand, three treatments-binomial, uniform, and unknown-are proposed between-subject. Halevy (2007) only proposes the last two treatments, and, more importantly, within-subject. With this design, he can examine the relation between attitude toward ambiguity and attitude toward reduction of compound (objective) lotteries, a question which is outside our scope of interest. Ahn et al. (2011) is also close to our experiment design. However, differently from their study, in each task of our experiment the asset contained in the portfolio is either safe or ambiguous. Moreover, we simplify the choice problem by limiting the choice set to only four possible portfolios and considering an ambiguous environment with only two states of nature.

The following is a discussion of our design against studies in the second group, i.e., as our study, those explicitly designed to operationalize $K M M$. Here, experimental results will also be compared.

Similarly to Chakravarty and Roy (2009), our study separates attitude toward risk from that toward ambiguity. However, their main purpose radically differs from ours. They investigate potential differences in subjects' behavior under uncertainty over gains versus uncertainty over losses, while in our experiment there are no negative outcomes. Moreover, we use portfolio choice questions and the BDM mechanism to 
elicit risk and ambiguity attitude, while they use the multiple price list method. As anticipated above, they find a positive correlation between risk attitude and ambiguity attitude in the domain of gains, while our results tend to lean toward a negative correlation.

Our experimental findings about the performance of $K M M$ are in line with Conte and Hey (2013): almost 9 out of 10 subjects in our experiment are classified as having a coherent-ambiguity attitude within the KMM model, and almost 8 out of 10 of them satisfy all theoretical predictions derived within that model. Coherent-ambiguityneutral subjects are those who have the highest percentage of compliance with $K M M$ predicted behavior. Recalling that for these subjects $K M M$ reduces to the (subjective) expected utility model, we can relate this result to the one found by Halevy (2007) about the higher ability of ambiguity-neutral subjects to reduce compound lotteries.

Further, we find that highly-risk-averse subjects are more frequently ambiguityloving than ambiguity-averse. This brings up the possible existence of a negative correlation between risk attitude and ambiguity attitude, as the one found by Andersen et al. (2009). It should be noticed that our experimental design only reveals the sign of the subject's (coherent)-ambiguity attitude, i.e., whether she is (coherent)-ambiguityaverse, neutral, or loving. Sorting all classified subjects in three groups according to this sign, we find significant differences in the distributions of the degree of risk aversion among the three groups. In particular, (coherent)-ambiguity-averse and (coherent)ambiguity neutral subjects on average have a low degree of risk aversion, while the vast majority of (coherent)-ambiguity-loving subjects is highly-risk-averse. This point requires a more thorough discussion, provided in the next section.

Finally, we find that the percentage of coherently-ambiguity-averse subjects is lower in the binomial than in the uniform and in the unknown treatment. However, only the difference between the binomial and the unknown treatment is statistically significant. This result is partially in line with Abdellaoui et al. (2011), for the part where they state that attitude toward ambiguity and attitude toward compound risks are related but distinct, with this relationship being quite sensitive to the type of compound risks considered. They define as compound risk those decision tasks where the second-order probability distribution over one-stage lotteries is objective: both our binomial and our uniform treatment belong to this category. In our study too, therefore, the relation between what they call 'ambiguity' (our unknown treatment) and what they call 'compound risk' depends on the type of compound risk considered, e.g., binomial or uniform. However, there are two crucial differences between our experimental design and the one implemented in Abdellaoui et al. (2011). First, although they analyze the same uniform case as in our study, they do not treat the binomial case, focusing instead on the hypergeometric case. ${ }^{57}$ Second and more importantly, as Halevy (2007), they have a within-subject design, while in our study a subject only participates in one treatment, hence facing only one of the second-order probability distributions (binomial, uniform, or unknown) that we generate so as to create ambiguity.

\footnotetext{
57 Their actual finding is that the hypergeometric case is the one having the strongest relationship with ambiguity attitude.
} 


\section{Conclusion}

This study indicates ways to identify two features of smooth ambiguity attitude à la $K M M$ : value-ambiguity attitude and choice-ambiguity attitude. A value-ambiguityaverse subject values an ambiguous lottery less than its unambiguous equivalent with the same mean probabilities. A choice-ambiguity-averse subject always reduces her demand for the risky asset when the distribution of outcomes becomes ambiguous.

We elicit these two attitudes in a series of experimental decision tasks designed in order to match the main insights of the $K M M$ model. Our decision tasks are parameterized so that a value-ambiguity-averse (-loving) subject should necessarily behave as a choice-ambiguity-averse (-loving) one, i.e., showing coherent-ambiguity aversion. Indeed, we find that $88 \%$ of our subjects (92/105) show a coherentambiguity attitude, independent of the treatment, i.e., the distribution of secondorder probabilities (binomial, uniform, or unknown). This result clearly indicates an equivalence between value-ambiguity aversion and choice-ambiguity aversion in subjects participating in our experiment. However, we do not find the same equivalence between strong value-ambiguity aversion and strong choice-ambiguity aversion.

A key contribution of this paper is the theoretical analysis of ambiguous tasks designed to evaluate the structure of risk and ambiguity attitudes within the $K M M$ model. In Sect. 3, we have provided two kinds of theoretical predictions: those holding independent of the subject's (coherent)-ambiguity attitude and those stating specific behavior in correspondence of a specific attitude to (coherent)-ambiguity. We find that the former are satisfied by more than $90 \%$ of our classified subjects (84/92), while the latter are complied with by more than $80 \%$ of classified subjects (74/92). Overall, a large number of classified subjects $(76 \%, 70 / 92)$ satisfy all our theoretically derived constraints. This remarkably high compliance of subjects' behavior with $K M M$ indirectly corroborates both our operational definition of (coherent)ambiguity attitude and our experimental design being a correct representation of the main features of $K M M$ in the laboratory. We do not find any significant correlation between compliance with $K M M$ and gender, age, level of education, and degree of risk attitude.

A secondary contribution of this paper concerns the analysis of possible relations between risk attitude and ambiguity attitude. We elicit risk attitude through the same two methods (portfolio choice and BDM) used to elicit the two features (respectively, choice and value) of coherent-ambiguity attitude. Risk-aversion orderings provided by the two methods are correlated. Relying on $C A R A$ or $C R R A$ orderings of risk aversion built through the first method, we find that more than $1 / 2$ of subjects may be classified as CARA and almost 3/4 as CRRA. Under both specifications, we find a negative correlation between the degree of risk aversion elicited in the unambiguous tasks and the fact of showing coherent-ambiguity aversion in the ambiguous tasks. However, this correlation is significant if we consider only choice-ambiguity attitude. More specifically, in our sample many coherent-ambiguity-averse subjects have a low degree of risk aversion, while the most part of coherent-ambiguity-loving subjects have a high degree of it (77\% of the coherent-ambiguity-loving subjects are in the last 
two quintiles of the $C A R A$ index distribution, and $73 \%$ are in the last two quintiles of the $C R R A$ index distribution).

A possible explanation for this apparently surprising result is as follows. This result may be linked to the riskiness of the second-stage lottery the subject chooses in the unambiguous task (that is the same she is assigned in the ambiguous task). The higher the degree of risk aversion, the less risky the chosen lottery in the unambiguous task, the lower the subject's value for this lottery, then, the lower the reduction of the subject's value for this lottery when the distribution of outcomes becomes ambiguous. The positive correlation between the riskiness of the lottery assigned in the most ambiguous task and both value-ambiguity aversion and choice-ambiguity aversion that we find in the data supports this explanation. However, this result is found in a between-subject design: we ask a subject to state her certainty equivalent under different levels of ambiguity of the setting, but always facing the same pair of (second-stage) lottery outcomes. One may test whether the effect found between-subject also holds when the same subject is asked to state her attitude toward ambiguity for different pairs of lottery outcomes. This point is left for further research.

The third contribution of this paper concerns the analysis of subjects' attitudes and decisions in ambiguous tasks with specific distributions of second-order probabilities. We find that the percentage of coherently-ambiguity-averse subjects is lower (though not significantly) in the binomial than in the uniform treatment. This was easily predictable, as the latter distribution of second-order probabilities is a mean-preserving spread of the former. We also verify the prediction of a significantly lower percentage of coherent-ambiguity-averse subjects in the binomial than in the unknown treatment: indeed the latter is intrinsically more ambiguous than the former. A surprising fact is the absence of a significant difference in the percentage of coherent-ambiguity-averse subjects between the uniform and the unknown treatment. This may lead to validate the assumption that subjective second-order probabilities may be thought as uniformly distributed when the subject is not given any information about the composition of the unknown urn.

Acknowledgments The research leading to these results has received funding from the European Research Council under the European Community's Seventh Framework Programme (FP7/2007-2013) Grant Agreement No. 230589. G. Attanasi gratefully acknowledges financial support by the Chair Georges Meyer in Mathematical Economics at Jean-Jacques Laffont Foundation (TSE). N. Pace gratefully acknowledges financial support by the Swiss\&Global and Fondazione Ca' Foscari. The authors gratefully thank Michèle Cohen, Sandrine Spaeter, Lorenzo Vantaggiato, participants at the 15th Conference on the Foundations and Applications of Utility, Risk and Decision Theory at Georgia State University in Atlanta, at the Workshop on New Developments in Decision Making under Uncertainty at Université de Cergy-Pontoise, and at a Cournot seminar at University of Strasbourg for their useful comments and suggestions.

\section{Appendix}

\subsection{Appendix A}

For each lottery $l_{t}^{j}=\left(\bar{x}_{t}^{j}, 0.5 ; \underline{x}_{t}^{j}, 0.5\right)$ in Table 2 , the expected value and the standard deviation are respectively equal to $E V=0.5(\bar{x}+\underline{x})$ and $\sigma=0.5(\bar{x}-\underline{x})$. The two 
Table 7 Risk attitude elicitation method: reinterpretation in terms of $E V, \sigma$, and $(d \sigma / d E V)$

\begin{tabular}{|c|c|c|c|c|c|c|c|c|c|c|c|c|}
\hline \multirow[t]{2}{*}{ Lottery } & \multicolumn{3}{|c|}{ Task $t=1$} & \multicolumn{3}{|c|}{ Task $t=2$} & \multicolumn{3}{|c|}{ Task $t=3$} & \multicolumn{3}{|c|}{ Task $t=4$} \\
\hline & $E V_{1}^{j}$ & $\sigma_{1}^{j}$ & $\frac{d \sigma_{1}^{j}}{d E V_{1}^{j}}$ & $E V_{2}^{j}$ & $\sigma_{2}^{j}$ & $\frac{d \sigma_{2}^{j}}{d E V_{2}^{j}}$ & $E V_{3}^{j}$ & $\sigma_{3}^{j}$ & $\frac{d \sigma_{3}^{j}}{d E V_{3}^{j}}$ & $E V_{4}^{j}$ & $\sigma_{4}^{j}$ & $\frac{d \sigma_{4}^{j}}{d E V_{4}}$ \\
\hline$j=A$ & 9.0 & 3.0 & 3.0 & 8.5 & 2.5 & 5.0 & 17.0 & 3.0 & 3.0 & 16.5 & 2.5 & 5.0 \\
\hline$j=B$ & 10.0 & 6.0 & 3.0 & 9.0 & 5.0 & 5.0 & 18.0 & 6.0 & 3.0 & 17.0 & 5.0 & 5.0 \\
\hline$j=C$ & 11.0 & 9.0 & 3.0 & 9.5 & 7.5 & 5.0 & 19.0 & 9.0 & 3.0 & 18.0 & 10.0 & 5.0 \\
\hline$j=D$ & 12.0 & 12.0 & 3.0 & 10.0 & 10.0 & 5.0 & 20.0 & 12.0 & 3.0 & 19.0 & 15.0 & 5.0 \\
\hline
\end{tabular}

lottery outcomes can be expressed in terms of the two moments, i.e., $\bar{x}=E V+\sigma$ and $x=E V-\sigma$. In Table 7, the set of lotteries in tasks 1-4 are classified in terms of the triple $\left(E V, \sigma, \frac{d \sigma}{d E V}\right)$, where the ratio $\frac{d \sigma}{d E V}$ is the same for all lotteries in the same task. In particular, it is $\frac{d \sigma}{d E V}=3$ in tasks 1 and 3 , and $\frac{d \sigma}{d E V}=5$ in tasks 2 and 4 .

\subsection{Appendix B}
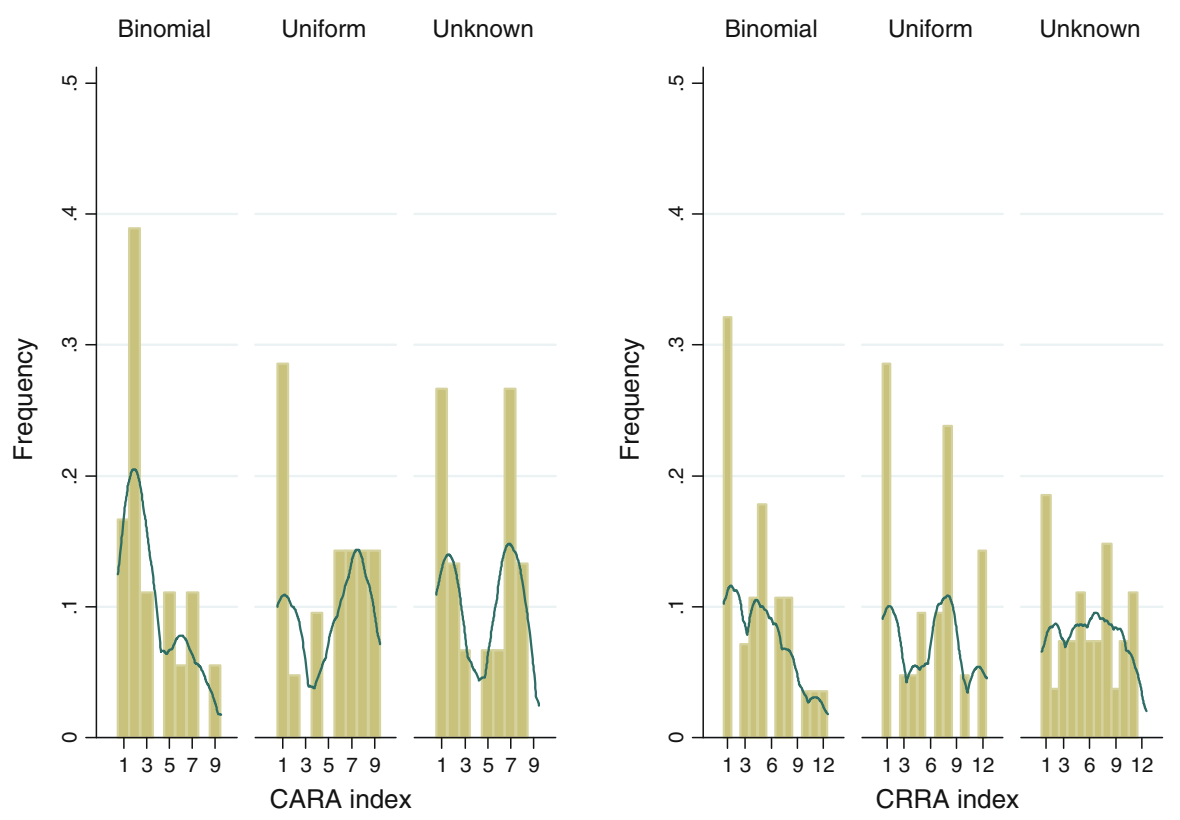

Fig. 8 Distribution of $C A R A$ and $C R R A$ index by treatment 


\subsection{Appendix C}
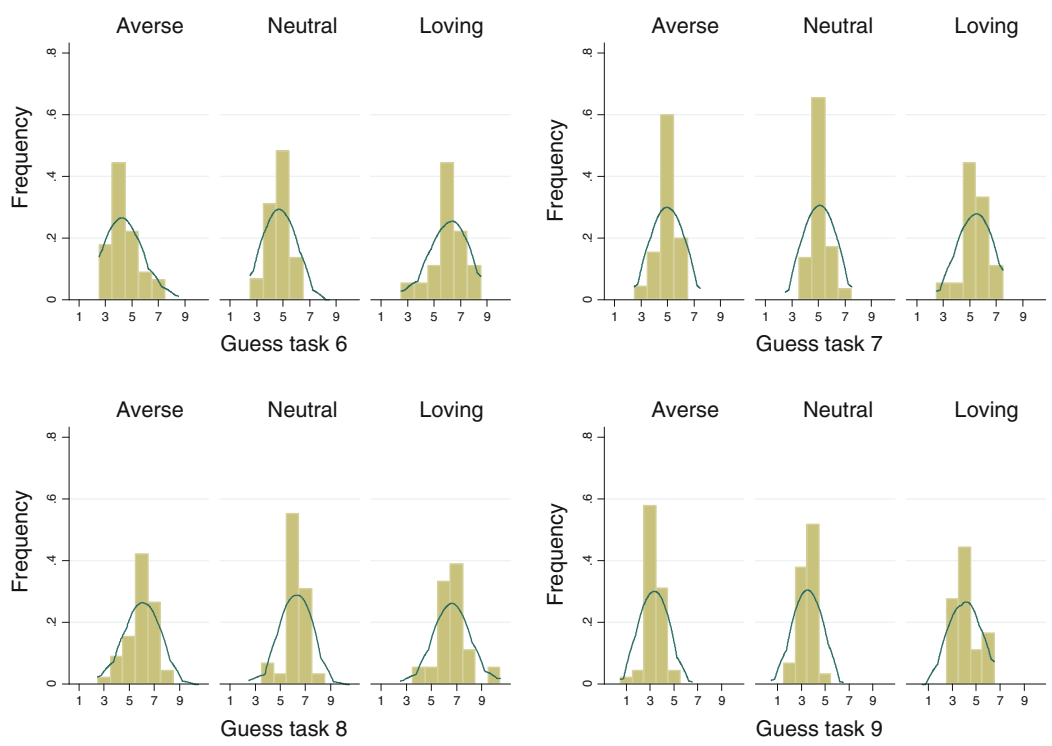

Fig. 9 Distribution of subjects' guess on the number of "winning balls" in tasks 6-9 by ambiguity attitude

\subsection{Appendix D}
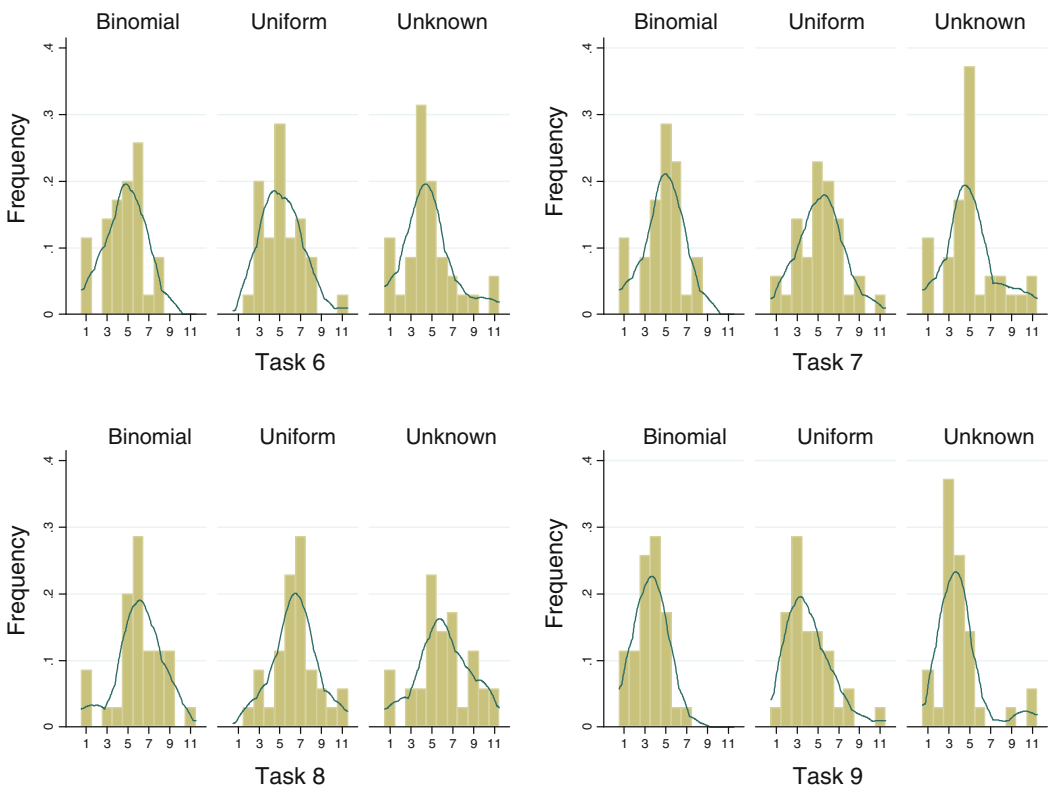

Fig. 10 Distribution of "normalized" $C E\left(L_{t}\right)$ in tasks $6-9$ by treatment 


\section{References}

Abdellaoui, M., Klibanoff, P., \& Placido, L. (2011). Ambiguity and compound risk attitudes: An experiment. Working paper. Northwestern University.

Ahn, D., Choi, S., Gale, D., \& Kariv, S. (2011). Estimating ambiguity aversion in a portfolio choice experiment. Working paper. University of California, Berkeley.

Andersen, S., Fountain, J., Harrison, G., \& Rutström, E. (2009). Estimating aversion to uncertainty. Working paper. University of Central Florida.

Arrow, K. (1964). The role of securities in the optimal allocation of risk bearing. Review of Economic Studies, 31, 91-96.

Attanasi, G., \& Montesano, A. (2012). The price for information about probabilities and its relation with risk and ambiguity. Theory and Decision, 73, 125-160.

Becker, G., DeGroot, M. H., \& Marschak, J. (1964). Measuring utility by a single response sequential method. Behavioral Science, 9, 226-232.

Becker, S. W., \& Brownson, F. O. (1964). What price ambiguity? Or the role of ambiguity in decision making. Journal of Political Economy, 72, 62-73.

Bossaerts, P., Ghirardato, P., Guarnaschelli, S., \& Zame, W. (2010). Prices and allocations in asset markets with heterogeneous attitudes towards ambiguity. Review of Financial Studies, 23, 1325-1359.

Camerer, C. (1995). Individual decision making. In J. Kagel \& A. Roth (Eds.), Handbook of experimental economics (pp. 587-703). Princeton University Press.

Camerer, C., \& Weber, M. (1992). Recent development in modeling preferences: Uncertainty and ambiguity. Journal of Risk and Uncertainty, 5, 325-370.

Chakravarty, S., \& Roy, J. (2009). Recursive expected utility and the separation of attitudes towards risk and ambiguity: An experimental study. Theory and Decision, 66, 199-228.

Cohen, M., Jaffray, J.-Y., \& Said, T. (1987). Experimental comparison of individual behavior under risk and under uncertainty for gains and for losses. Organizational Behavior and Human Decision Processes, 39 , $1-22$.

Cohen, M., Tallon, J. M., \& Vergnaud, J. C. (2011). An experimental investigation of imprecision attitude and its relation with risk attitude and impatience. Theory and Decision, 71, 81-109.

Conte, A., Hey, J. D. (2013). Assessing multiple prior models of behavior under ambiguity. Journal of Risk and Uncertainty, 46, 113-132.

Conte, A., Hey, J. D., \& Moffatt, P. G. (2011). Mixture models of choice under risk. Journal of Econometrics, $162,79-82$.

Cox J. C., Sadiraj V., \& Schmidt U. (2012). Paradoxes and mechanisms for choice under risk. Experimental Economics Center Working Paper Series 2012-08, Experimental Economics Center, Andrew Young School of Policy Studies, Georgia State University.

Di Mauro, C., \& Maffioletti, A. (2004). Attitudes to risk and attitudes to uncertainty: Experimental evidence. Applied Economics, 36, 357-372.

Ellsberg, D. (1961). Risk, ambiguity and the Savage axioms. Quarterly Journal of Economics, 75, 643-669.

Epstein, L. G. (2010). A paradox for the "smooth ambiguity" model of preference. Econometrica, 78, 2085-2099.

Fischbacher, U. (2007). z-Tree: Zurich toolbox for ready-made economic experiments. Experimental Economics, 10, 171-178.

Ghirardato, P. (2004). Defining ambiguity and ambiguity attitude. In I. Gilboa (Ed.), Uncertainty in economic theory: A collection of essays in honor of David Schmeidler's 65th birthday (pp. 36-45). London: Routledge.

Ghirardato, P., Maccheroni, F., \& Marinacci, M. (2004). Differentiating ambiguity and ambiguity attitude. Journal of Economic Theory, 118, 133-173.

Gollier, C. (2001). Should we beware of the precautionary principle? Economic Policy, 16, 301-328.

Gollier C. (2012). Optimal insurance design of ambiguous risks. IDEI Working Paper no. 718.

Halevy, Y. (2007). Ellsberg revisited: An experimental study. Econometrica, 75, 503-536.

Harrison, G. W. (2011). Experimental methods and the welfare evaluation of policy lotteries. European Review of Agricultural Economics, 38, 335-360.

Harrison, G. W., Johnson, E., McInnes, M. M., \& Rutström, E. E. (2005). Risk aversion and incentive effects: Comment. American Economic Review, 95, 897-901. 
Harrison, G. W., Martinez-Correa, J., \& Swarthout J. T. (2012). Reduction of compound lotteries with objective probabilities: Theory and evidence. Working Paper 2012-05, Center for the Economic Analysis of Risk, Robinson College of Business, Georgia State University.

Harrison, G. W., \& Rutström, E. E. (2008). Risk aversion in the laboratory. In G. W. Harrison \& J. Cox (Eds.), Risk aversion in experiments (pp. 41-196). Bradford, UK: JAI Press.

Harrison, G. W., \& Rutström, E. (2009). Expected utility theory and prospect theory: One wedding and a decent funeral. Experimental Economics, 12, 133-158.

Harrison G. W., \& Swarthout T. (2012). The independence axiom and the bipolar behaviorist. Experimental Economics Center Working Paper Series 2012-01, Experimental Economics Center, Andrew Young School of Policy Studies, Georgia State University.

Hey, J. D., \& Lee, J. (2005). Do subjects separate (or are they sophisticated)? Experimental Economics, 8, 233-265.

Hey, J. D., Lotito, G., \& Maffioletti, A. (2010). The descriptive and predictive adequacy of theories of decision making under uncertainty/ambiguity. Journal of Risk and Uncertainty, 41, 81-111.

Hey, J. D., \& Pace, N. (2014). The explanatory and predictive power of non two-stage-probability theories of decision making under ambiguity. Journal of Risk and Uncertainty (forthcoming).

Holt, C. A. (1986). Preference reversal and the independence axiom. American Economic Review, 76, $508-515$.

Holt, C. A., \& Laury, S. K. (2002). Risk aversion and incentive effects. American Economic Review, 92, $1644-1655$.

Holt, C. A., \& Laury, S. K. (2005). Risk aversion and incentive effects: New data without order effects. American Economic Review, 95, 902-912.

Kadane J. B. (1992). Healthy skepticism as an expected-utility explanation of the phenomena of Allais and Ellsberg. In: Geweke J. (Ed.), Decision making under risk and uncertainty: New models and empirical findings. Boston: Kluwer. (And in Theory and Decision, 32, 57-64).

Karni, E., \& Safra, Z. (1987). 'Preference reversal' and the observability of preferences by experimental methods. Econometrica, 55, 675-685.

Klibanoff, P., Marinacci, M., \& Mukerji, S. (2005). A smooth model of decision making under ambiguity. Econometrica, 73, 1849-1892.

Klibanoff, P., Marinacci, M., \& Mukerji, S. (2012). On the smooth ambiguity model: A reply. Econometrica, 80, 1303-1321.

Knight, F. (1921). Risk, uncertainty, and profit. Boston: Houghton Mifflin.

Lauriola, M., \& Levin, I. P. (2001). Relating individual differences in attitude toward ambiguity and risky choices. Journal of Behavioral Decision Making, 14, 107-122.

Lee, J. (2008). The effect of the background risk in a simple chance improving decision model. The Journal of Risk and Uncertainty, 36, 19-41.

Machina, M. (2004). Almost objective uncertainty. Economic Theory, 24, 1-54.

Plott, C. R., \& Zeiler, K. (2005). The willingness to pay-willingness to accept gap, the "endowment effect", subject misconceptions, and experimental procedures for eliciting valuations. American Economic Review, 95, 530-545.

Pratt, J. W. (1964). Risk aversion in the small in the large. Econometrica, 32, 122-136.

Quiggin, J. (2007). Ambiguity and the value of information: An almost-objective events analysis. Economic Theory, 30, 409-414.

Savage, L. J. (1954). The foundations of statistics. New York: Wiley.

Schneeweiss, H. (1973). The Ellsberg Paradox from the point of view of game theory. Inference and Decision, 1, 65-78.

Snow, A. (2010). Ambiguity and the value of information. Journal of Risk and Uncertainty, 40, $133-145$.

Starmer, C., \& Sugden, R. (1991). Does the random-lottery incentive system elicit true preferences? An experimental investigation. American Economic Review, 81, 971-978.

Tversky, A., \& Kahneman, D. (1992). Advances in prospect theory: Cumulative pepresentation of uncertainty. Journal of Risk and Uncertainty, 5, 297-323. 